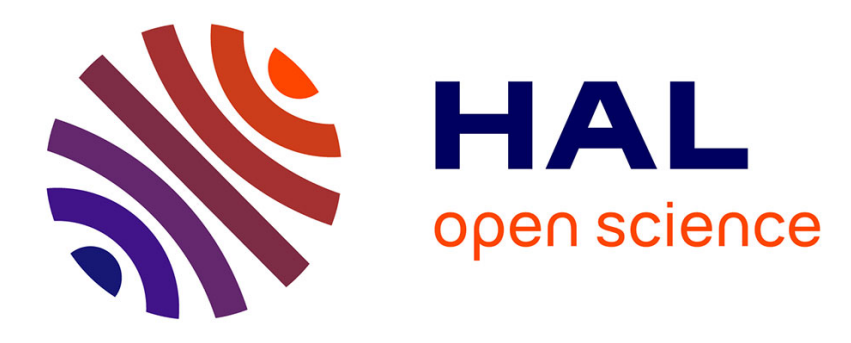

\title{
Mathematical morphology on hypergraphs, application to similarity and positive kernel.
}

Isabelle Bloch, Alain Bretto

\section{To cite this version:}

Isabelle Bloch, Alain Bretto. Mathematical morphology on hypergraphs, application to similarity and positive kernel.. Computer Vision and Image Understanding, 2013, http://dx.doi.org/10.1016/j.cviu.2012.10.013. 10.1016/j.cviu.2012.10.013 . hal-01010406

\section{HAL Id: hal-01010406 https://hal.science/hal-01010406}

Submitted on 19 Jun 2014

HAL is a multi-disciplinary open access archive for the deposit and dissemination of scientific research documents, whether they are published or not. The documents may come from teaching and research institutions in France or abroad, or from public or private research centers.
L'archive ouverte pluridisciplinaire HAL, est destinée au dépôt et à la diffusion de documents scientifiques de niveau recherche, publiés ou non, émanant des établissements d'enseignement et de recherche français ou étrangers, des laboratoires publics ou privés. 


\title{
Mathematical morphology on hypergraphs, application to similarity and positive kernel
}

\author{
Isabelle Bloch $^{\mathrm{a}, *}$, Alain Bretto ${ }^{\mathrm{b}}$ \\ a Institut Mines-Telecom, Telecom ParisTech, CNRS LTCI, Paris, France \\ ${ }^{\mathrm{b}}$ Université de Basse Normandie Caen, Greyc Cnrs-Umr 6072, Caen, France
}

\begin{abstract}
A B S T R A C T
The focus of this article is to develop mathematical morphology on hypergraphs. To this aim, we define lattice structures on hypergraphs on which we build mathematical morphology operators. We show some relations between these operators and the hypergraph structure, considering in particular transversals and duality notions. Then, as another contribution, we show how mathematical morphology can be used for classification or matching problems on data represented by hypergraphs: thanks to dilation operators, we define a similarity measure between hypergraphs, and we show that it is a kernel. A distance is finally introduced using this similarity notion.
\end{abstract}

\section{Introduction}

Mathematical morphology, which appeared in the 1960s, is a theory of non-linear information processing. It is now widely used in image analysis and pattern recognition, with applications in many domains [1-4]. In its deterministic part, mathematical morphology relies on the algebraic framework of complete lattices and increasing operators are defined from the core notion of adjunction $[5-8]^{1}$

Hypergraphs were also introduced in the 1960 s as a generalization of graphs [10], where edges become hyperedges and can connect more than two vertices, which thus offers an increased representation and expressivity power. Since then, they have been intensively studied, and a substantial amount of research in graph theory continues in hypergraph theory. They have shown their interest in various fields such as computer science, game theory, databases, data mining, optimization [11], image processing, segmentation, and retrieval [12-16]. The theory of hypergraphs deals with combinatorial set systems. So we can say that mathematical morphology and hypergraphs have common bases, which deserve to be further explored.

In order to deal with structured information, mathematical morphology has been developed on graphs [17-20] and on simpli-

\footnotetext{
* Corresponding author.

E-mail addresses: isabelle.bloch@telecom-paristech.fr (I. Bloch), alain.bretto@ info.unicaen.fr (A. Bretto).

1 Actually semi-lattices or even partially ordered sets would be sufficient [9], together with the adjunction principle, but we will restrict ourselves to the most classical framework of complete lattices in this paper.
}

cial complexes [21], and it is based on adjunctions defined on vertices and edges of (weighted) graphs. Specific forms of morphological operators involve the notion of structuring element, which represents a neighborhood or a binary relation between elements of the underlying space. Actually the notion of neighborhood is central to define dilations and erosions on graphs, and is most often defined from the binary relations between graph vertices defined by the edges. However such binary relations can be restrictive and relations of arity larger than two are needed in many domains (databases, pattern recognition, data mining, recommendation systems, etc.). Therefore, introducing mathematical morphology on hypergraphs seems relevant, and has not been addressed before to the best of our knowledge. The main objective of this paper is therefore to define mathematical morphology operators on hypergraphs, based on appropriate lattice structures.

Comparing two structures (algebraic, combinatorial, etc.) can be done via the notion of isomorphism. This notion is however often too strong to find just the "concordance" between the structures. Moreover, to date there is no efficient algorithm to compute an isomorphism between two graphs or two hypergraphs. Measures of similarity between graphs play a fundamental role in many varied fields. These concepts have been extensively studied because the applications are countless [22]. Specifically, we find similarity measures on graphs in chemistry, clustering, computer science, World Wide Web network, etc. A very popular similarity tool consists of kernels which can encode prior knowledge about objects in machine learning [23]. Kernels can be viewed as a kind of inner products. There are a lot of types of kernels, e.g. Gaussian kernels, Fisher kernels, etc., which describe different notions of similarity between objects. Little work has been done on the similarities 

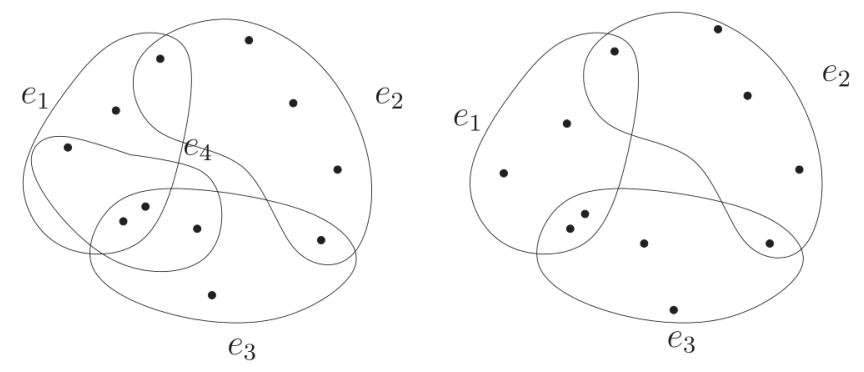

Fig. 1. Two hypergraphs $\left(V, E_{1}\right),\left(V, E_{2}\right)$, defined on the same set of vertices Hyperedges are displayed as sets of vertices. Three hyperedges are the same, and $E_{1}$ contains an additional hyperedge $\left(e_{4}\right)$ with respect to $E_{2}$. A quite high similarity between the two hypergraphs should then be expected.

between hypergraphs. Therefore, as another contribution, we propose original ways of comparing hypergraphs, based on morphological dilations. This should allow us to answer questions such as "are the two hypergraphs illustrated in Fig. 1 similar? to which extent are they similar?", and therefore constitutes a concrete application of the morphological operators introduced on hypergraphs.

We denote the universe of hypergraphs as $\mathcal{H}=(\mathcal{V}, \mathcal{E})$ with $\mathcal{V}$ the set of vertices and $\mathcal{E}$ the set of hyperedges. The powersets of $\mathcal{V}$ and $\mathcal{E}$ are denoted by $\mathcal{P}(\mathcal{V})$ and $\mathcal{P}(\mathcal{E})$, respectively. We denote a hypergraph by $H=(V, E)$ with $V \subseteq \mathcal{V}$ and $E \subseteq \mathcal{E}$.

After introducing some more notations and basic concepts related to hypergraphs in Section 2, we define in Section 3 complete lattice structures $(\mathcal{T}, \preceq)$ on the hypergraphs of $\mathcal{H}$, with $\preceq$ a partial ordering on $\mathcal{T}$. Mathematical morphology operators on hypergraphs are then defined in Section 4. Sections 3 and 4 therefore constitute the core of the proposed framework. In the next sections, we show how to use this framework for different hypergraph concepts. In Section 5, we consider dual hypergraphs and establish some links between morphological dilations and hypergraph duality concepts. In Section 6 we propose a similarity notion between hypergraphs based on dilations. After introducing this concept we show its relevance, and we associate to this similarity a positive kernel. Section 7 is devoted to a distance on hypergraphs. A simple example on an image represented by a hypergraph associated with a simplicial complex is finally provided in Section 8. This paper extends our preliminary work in [24]. It shows the close connections between the theories of hypergraphs and mathematical morphology, which derive from their common bases relying on set theory and lattices in particular. Therefore the extension we propose in this paper can be considered as a natural follow-up of previous works associating mathematical morphology with other structures such as graphs and simplicial complexes. We also establish and clarify the relations between the morphological operators on hypergraphs and some useful hypergraph properties such as duality, transversals, similarity, and matching.

\section{Basic concepts on hypergraphs}

In this section, we recall some definitions which are used in the next sections, using similar notations and concepts as in [10]. The reader is referred to this reference for details.

A hypergraph $H$ denoted by $H=\left(V, E=\left(e_{i}\right)_{i \in I}\right)$ is defined as a finite set $V$ of vertices and a finite family $\left(e_{i}\right)_{i \in I}$, (where $I$ is a finite set of indices) of non-empty subsets of $V$ called hyperedges. ${ }^{2}$ We generally consider that hyperedges are non-empty, except when needed for

\footnotetext{
${ }^{2}$ Note that definitions can be extended to the infinite case, but this is not considered in this paper and we restrict ourselves to the finite case.
}

technical reasons. We also consider that $H$ is without repeated hyperedge, i.e. every hyperedge is uniquely represented in $E$.

Let $\left(e_{j}\right)_{j \in \text {, }}$, with $J \subseteq I$ be a sub-family of hyperedges of $E$. The set of vertices belonging to these hyperedges is denoted by $v\left(\cup_{j \in j} e_{j}\right)$ or $\cup_{j \in J} v\left(e_{j}\right)$, and $v(e)$ denotes the set of vertices forming the hyperedge $e$. If $\cup_{i \in I} u\left(e_{i}\right)=V$, the hypergraph is without isolated vertex (a vertex $x$ is isolated if $x \in V \backslash \cup_{i \in I} v\left(e_{i}\right)$ ). Note that if there is a hyperedge $e=\{x\}$ in $E$, then $x$ is not isolated. Hence the notion of isolated vertex in a hypergraph is not directly linked to the one of isolated point in a graph, i.e. a connected component reduced to one point. By definition the empty hypergraph is the hypergraph $H_{\emptyset}$ such that $V=\emptyset$ and $E=\emptyset$.

Let $H=\left(V,\left(e_{i}\right)_{i \in I}\right)$ be a hypergraph.

- The partial hypergraph $H^{\prime}$ of $H$ generated by $J \subseteq I$ is the hypergraph $\left(V,\left(e_{j}\right)_{j \in J}\right)$.

- Given a subset $V^{\prime} \subseteq V$, a subhypergraph $H^{\prime}$ is the partial hypergraph $H^{\prime}=\left(V^{\prime},\left\{e_{i}, i \in I \mid v\left(e_{i}\right) \subseteq V^{\prime}\right\}\right)$.

- The induced subhypergraph $H\left(V^{\prime}\right)$ of $H$ with $V^{\prime} \subseteq V$ is the hypergraph defined as $H\left(V^{\prime}\right)=\left(V^{\prime}, E^{\prime}\right)$ with $E^{\prime}=\left\{e^{\prime}=\left\{v(e) \cap V^{\prime}\right\} \mid e \in E\right.$ and $\left.v(e) \cap V^{\prime} \neq \emptyset\right\}$. Note that if $V^{\prime}=V$ and hypergraphs are considered without empty hyperedges, then $H\left(V^{\prime}\right)=H(V)=H$.

Without loss of generality we can suppose that the empty hypergraph, $H_{\emptyset}=(\emptyset, \emptyset)$ is a partial hypergraph, (resp. (induced) subhypergraph) of any hypergraph.

The star centered at $x$, for $x \in V$, is the set of hyperedges containing $x$, denoted by $H(x)$. The value $d(x)=|H(x)|$ is the degree of $x$.

The rank of $H$ is the maximal cardinality of a hyperedge. A hypergraph is linear if $\left|e_{i} \cap e_{j}\right| \leqslant 1$ for $i \neq j$. A loop is a hyperedge with a cardinality equal to one. A simple hypergraph is a hypergraph $H=\left(V, E=\left(e_{i}\right)_{i \in I}\right)$ such that: $e_{i} \subseteq e_{j} \Rightarrow i=j$.

The dual $H^{*}$ of a hypergraph without empty hyperedge and without isolated vertex $H$ is a hypergraph whose set of vertices is isomorphic (denoted $\simeq$ ) to the set of hyperedges of $H$, and whose hyperedges are given by $X_{1}, X_{2}, \ldots, X_{n}$ where $X_{j}=\left\{e_{i} \mid x_{j} \in v\left(e_{i}\right)\right\}$ for each vertex $x_{j}$ of $H$. The transpose $A^{t}$ of the incidence matrix $A=\left(\left(a_{i j}\right)\right)_{i \in\{1, \ldots, \mid V\}, j \in I}$, where $V$ is the set of vertices and $I$ is the index set of the set of hyperedges $E$, of a hypergraph $H$ (i.e. $a_{i j}=1$ iff vertex $i$ belongs to hyperedge $j)$ is the incidence matrix of $H^{*}=\left(V^{*} \simeq E\right.$, $\left.E^{*} \simeq(H(x))_{x \in V}\right)$ : for $v_{j}^{*} \in V^{*}$ and $e_{i}^{*} \in E^{*}, v_{j}^{*} \in e_{i}^{*}$ if and only if $a_{i j}=1$, and $\left(H^{*}\right)^{*}=H$. Note that a hypergraph can be equivalently defined as a family of hyperedges on a set of vertices, or as an incidence matrix. $^{3}$

A hypergraph $H=(V, E)$ is isomorphic to a hypergraph $H^{\prime}=\left(V^{\prime}, E^{\prime}\right)$ $\left(H \simeq H^{\prime}\right)$, if there exist a bijection $f: V \rightarrow V^{\prime}$ and a bijection $\pi: I \rightarrow J$, where $I$ and $J$ are the index sets of $E$ and $E^{\prime}$, respectively (i.e. $\left.E=\left(e_{i}\right)_{i \in I}, E^{\prime}=\left(e_{j}^{\prime}\right)_{j \in I}\right)$, which induce a bijection: $g: E \rightarrow E^{\prime}$ such that: $g\left(e_{i}\right)=e_{\pi(i)}^{\prime}$, for all $e_{i} \in E$ and $e_{\pi(i)}^{\prime} \in E^{\prime}$. The mapping $f$ is then called isomorphism of hypergraphs. Note that $H \simeq H^{\prime}$ if and only if $H^{*} \simeq H^{*}$

Let $H=(V, E)$ be a hypergraph, $E=\left(e_{1}, e_{2}, \ldots, e_{m}\right)$. A path $P$ in $H$ from $x_{i_{1}}$ to $x_{i_{s+1}}$ is an alternated vertex-hyperedge sequence $x_{i_{1}}, e_{i_{1}}, x_{i_{2}}, e_{i_{2}}, \ldots, x_{i_{s}}, e_{i_{s}}, x_{i_{s+1}} \quad$ such that $\left\{x_{i_{k}}, x_{i_{k+1}}\right\} \subseteq v\left(e_{i_{k}}\right),(k=$ $1,2, \ldots, s)$ and $x_{i_{k}} \neq x_{i_{i}}, e_{i_{k}} \neq e_{i_{j}}\left(i_{k} \neq i_{j}\right)$, where $s$ is called the length of path $P^{4}$ The distance between vertices $x$ and $y, d(x, y)$ is the

\footnotetext{
${ }^{3}$ Note that the notion of bipartite graph could also be used, since a bipartite graph can be associated with any hypergraph, where the set of vertices is the union of $V$ and $E$ and the set of edges is built from the pairs $(x, e), x \in V, e \in E$, such that $x \in v(e)$. However given a bipartite graph, two hypergraphs can be associated with it, one being the dual of the other. Therefore we stay within the framework of hypergraphs in this paper.

4 Note that the hyperedges $e_{i_{k}}$ and $e_{i_{k+1}}$ have necessarily at least $x_{i_{k+1}}$ as a common vertex. The definition of a path as an alternated vertex-hyperedge sequence allows defining precisely how the path goes from one hyperedge to the next one, i.e. through which vertex.
} 
minimum length among those of all paths which connect $x$ and $y$. If for each pair of vertices $\{x, y\}$ there is a path from $x$ to $y$, the hypergraph $H$ is said connected. Moreover, the relation $\mathcal{R}$ defined on $V \times V$ by:

$x \mathcal{R} y \Longleftrightarrow$ there is a path from $x$ to $y$ or $x=y$

is an equivalence relation. The equivalence classes are the connected components of the hypergraph.

\section{Lattice structures on hypergraphs}

In this section we define a few lattices on hypergraphs, as the basic algebraic structures on which mathematical morphology operators are then defined.

A lattice on the set of vertices can be simply defined by $\mathcal{T}=(\mathcal{P}(\mathcal{V}), \subseteq)$. This is the classical lattice defined on the powerset of a set, with the standard set inclusion as partial ordering. It is a complete lattice. However it is not really interesting here since it does not say anything on the structure of the hypergraph. Similarly, a lattice on the set of hyperedges can be defined by $\mathcal{T}=(\mathcal{P}(\mathcal{E}), \subseteq)$. Again it is a classical complete lattice on the powerset of a set and classical results can be used directly.

A more interesting lattice can be defined, based on closed sets of vertices, involving the stars of vertices. Let $H=(V, E)$ be a hypergraph and let $V^{\prime} \subseteq V$. We say that $V^{\prime}$ is a closed set if $\forall(x, y) \in V^{2}$, $v(H(x) \cap H(y)) \subseteq V^{\prime}$. We denote by $\mathcal{C}(H)$ the family of closed sets with the empty set.

Proposition 1. The structure $(\mathcal{C}(H), \subseteq)$ is a complete lattice. The infimum is $\wedge=\cap$ and the supremum is: $\forall\left(V^{\prime}, V^{\prime \prime}\right) \in \mathcal{C}(H)^{2}$, $V^{\prime} \vee V^{\prime \prime}=\cap\left\{V^{\prime \prime \prime} \in \mathcal{C}(H) \mid V^{\prime} \cup V^{\prime \prime} \subseteq V^{\prime \prime \prime}\right\}$, i.e. the intersection of all closed sets containing $V^{\prime} \cup V^{\prime \prime}$, and its extension to any family. The smallest element is $\emptyset$ and the largest element is $V$. Note that $\mathcal{C}(H)$ is a Moore family [25].

Proof. It is easy to show that $\subseteq$ is a partial ordering on $\mathcal{C}(H)$. The intersection of closed sets is a closed set. Let us show this for two closed sets $V^{\prime}$ and $V^{\prime \prime}$. Let $(x, y) \in\left(V^{\prime} \cap V^{\prime \prime}\right)^{2}$. Since $(x, y) \in V^{2}$, we have $v(H(x) \cap H(y)) \subseteq V^{\prime}$. Similarly we have $v(H(x) \cap H(y)) \subseteq V^{\prime \prime}$, and thus $v(H(x) \cap H(y)) \subseteq V^{\prime} \cap V^{\prime \prime}$. It is also easy to show that $V^{\prime} \cap V^{\prime \prime}$ is the largest lower bound. This extends directly to any family. For the supremum, the proposed formula is the lowest upper bound by construction. Unfortunately we do not have a more explicit formula. Hence $(\mathcal{C}(H), \subseteq)$ is a complete lattice. The smallest element is $\wedge \mathcal{C}(H)=\emptyset$ and the largest element is $\bigvee \mathcal{C}(H)=V$.

Note that closed sets are not intended in the classical topological sense here. In particular, while the union of closed sets in a topological space is closed, this is not the case here. For example, let us consider $V=\left\{x_{1}, \ldots, x_{6}\right\}$ and $E=\left\{e_{1}, e_{2}, e_{3}\right\}$ with $v\left(e_{1}\right)=\left\{x_{1}, x_{2}\right.$,$\left.x_{3}\right\}, v\left(e_{2}\right)=\left\{x_{3}, x_{4}, x_{5}\right\}$, and $v\left(e_{3}\right)=\left\{x_{5}, x_{6}\right\}$. It is easy to check that $v\left(e_{1}\right)$ and $v\left(e_{3}\right)$ are closed sets. However, $V^{\prime}=v\left(e_{1}\right) \cup v\left(e_{3}\right)$ is not. In particular we have $H\left(x_{3}\right) \cap H\left(x_{5}\right)=\left\{e_{2}\right\}$, but $v\left(e_{2}\right) \nsubseteq V^{\prime}\left(x_{4} \in v\left(e_{2}\right)\right.$ but $\left.x_{4} \notin V^{\prime}\right)$. Note also that a closed set is not necessarily a connected component. Let us consider again the previous example, but now with $v\left(e_{2}\right)=x_{4}$. The subsets $v\left(e_{1}\right)$ and $v\left(e_{3}\right)$ are still closed, and now so is $V^{\prime}=\vartheta\left(e_{1}\right) \cup v\left(e_{3}\right)$ (this time $\left.H\left(x_{3}\right) \cap H\left(x_{5}\right)=\emptyset \subseteq V^{\prime}\right)$. But $V^{\prime}$ is not a connected component.

Lattices on the hypergraphs themselves will now allow us to better account for the whole structural information encoded in hypergraphs, considering both vertices and hyperedges in the definition of the lattice structure.

The simplest idea is to consider the inclusion on the powerset of vertices and edges, respectively. Other ideas could be to define a partial ordering based on the notions of induced sub-hypergraph, partial hypergraph and sub-hypergraph. These are detailed in the next subsections.

In all cases, $\mathcal{T}$ is defined as:

$H=(V, E) \in \mathcal{T} \Longleftrightarrow\left\{\begin{array}{l}V \subseteq \mathcal{V} \\ E \subseteq \mathcal{E} \\ \{x \in \mathcal{V} \mid \exists e \in E, x \in v(e)\} \subseteq V\end{array}\right.$

The last condition ensures that $H$ is actually a hypergraph, where the hyperedges are sets of vertices of $V$, and can be equivalently written as $\forall e \in E, v(e) \subseteq V$. There is no equivalent restriction on $V$ if isolated vertices are accepted.

\subsection{Partial ordering based on the inclusion on the powersets of vertices and hyperedges}

The first definition we propose relies on an inclusion relation on both vertices and hyperedges.

\section{Definition 1.}

$\forall\left(H_{1}, H_{2}\right) \in \mathcal{T}^{2}, H_{1}=\left(V_{1}, E_{1}\right), H_{2}=\left(V_{2}, E_{2}\right), H_{1} \preceq H_{2} \Longleftrightarrow\left\{\begin{array}{l}V_{1} \subseteq V_{2} \\ E_{1} \subseteq E_{2}\end{array}\right.$

This definition is similar to the one used in [17] for graphs. Moreover, it reduces to the definition on graphs in the particular case where hyperedges contain exactly two vertices, i.e. are edges of a graph.

Proposition 2. The following properties hold:

- $\preceq$ defines a partial ordering on $\mathcal{T}$.

- The infimum is: $H_{1} \wedge H_{2}=\left(V_{1} \cap V_{2}, E_{1} \cap E_{2}\right)$, and for any family $\left(H_{i}\right): \bigwedge_{i} H_{i}=\left(\bigcap_{i} V_{i}, \bigcap_{i} E_{i}\right)$.

- The supremum is: $H_{1} \vee H_{2}=\left(V_{1} \cup V_{2}, E_{1} \cup E_{2}\right)$, and for any family $\left(H_{i}\right): \bigvee_{i} H_{i}=\left(\bigcup_{i} V_{i}, \bigcup_{i} E_{i}\right)$

- $(\mathcal{T}, \preceq)$ is a complete lattice, which is moreover sup-generated. Its smallest element is $H_{\emptyset}=(\emptyset, \emptyset)$ and its largest element is $\mathcal{H}=(\mathcal{V}, \mathcal{E})$. We have $\bigvee \emptyset=\wedge \mathcal{T}=H_{\emptyset}$ and $\wedge \emptyset=\bigvee \mathcal{T}=\mathcal{H}$

Proof. Let $H_{1}=\left(V_{1}, E_{1}\right)$ and $H_{2}=\left(V_{2}, E_{2}\right)$ be any hypergraphs in $\mathcal{T}$.

Let us first take $H_{2}=H_{1}$. Then $V_{1}=V_{2}$ and $E_{1}=E_{2}$ and a fortiori the inclusions hold, i.e. $\preceq$ is reflexive. If $H_{1} \preceq H_{2}$ and $H_{2} \preceq H_{1}$ then $V_{1} \subseteq V_{2} \subseteq V_{1}$, hence $V_{1}=V_{2}$. Similarly $E_{1}=E_{2}$. Thus $H_{1}=H_{2}$ and $\preceq$ is antisymmetric. If $H_{1} \preceq H_{2}$ and $H_{2} \preceq H_{3}$ then $V_{1} \subseteq V_{2} \subseteq V_{3}$. Similarly $E_{1} \subseteq E_{3}$ and $\preceq$ is transitive. It is therefore a partial ordering (which consists of classical inclusion on the subset of vertices on the one hand and on the subset of hyperedges on the other hand, hence the very direct proof).

Let us now consider $H=\left(V_{1} \cap V_{2}, E_{1} \cap E_{2}\right)$. We have $H \in \mathcal{T}, H \preceq H_{1}$ and $H \preceq H_{2}$. Let us now assume that there is a hypergraph $H_{3}=\left(V_{3}, E_{3}\right) \in \mathcal{T}$ such that $H_{3} \preceq H_{1}$ and $H_{3} \preceq H_{2}$. We have then $V_{3} \subseteq V_{1}, V_{3} \subseteq V_{2}$ and $V_{3} \subseteq V_{1} \cap V_{2}$. Similarly $E_{3} \subseteq E_{1}$ $\cap E_{2}$. Thus $H_{3} \preceq H$, and $H$ is therefore the largest lower bound: $H=H_{1} \wedge H_{2}$. The proof directly extends to any family $\left(H_{i}\right)$. The proof for the supremum is similar. Finally $(\mathcal{T}, \preceq)$ is a poset such that every family has a least upper bound and a largest lower bound. It is therefore a complete lattice. Any $H=(V, E)$ can be expressed as $H=\left(\cup_{x \in V}\{x\}, \cup_{e \in E}\{e\}\right)$. A canonical decomposition will be detailed in Section 4.2, as: $H=\left(\vee_{e \in E}(v(e),\{e\})\right) \vee\left(\vee_{x \in V \backslash \cup_{e \in E} v(e)}(\{x\}, \emptyset)\right)$. The smallest element is $\Lambda \mathcal{T}=\left(\cap_{V \in \mathcal{V}} V, \cap_{E \in \mathcal{E}} E\right)=(\emptyset, \emptyset)=H_{\emptyset}$. The largest element is $\bigvee \mathcal{T}=\left(\cup_{V \in \mathcal{V}} V, \cup_{E \in \mathcal{E}} E\right)=(\mathcal{V}, \mathcal{E})=\mathcal{H}$.

Note that it is not complemented (in order to have $E \cup E^{c}=\mathcal{E}$, we would have to consider in $E^{c}$ all hyperedges that are not in $E$, including those which have vertices both in $V$ and in $V^{c}$, so $\left(V^{c}, E^{c}\right)$ would not be a hypergraph in $\mathcal{T}$ ). 
A restriction of Definition 1 can be derived from the natural ordering produced by the notion of partial hypergraph.

\section{Definition 2.}

$\forall\left(H_{1}, H_{2}\right) \in \mathcal{T}^{2}, H_{1}{ }_{p} H_{2} \Longleftrightarrow\left\{\begin{array}{l}V_{1}=V_{2} \\ E_{1} \subseteq E_{2}\end{array}\right.$

Proposition 3. $\preceq_{p}$ is a partial ordering on $\mathcal{T}$, and $\left(\mathcal{T}_{V}, \preceq_{p}\right)$ is a complete lattice, where $\mathcal{T}_{V}$ denotes the set of hypergraphs with $V$ as set of vertices.

Proof. The proof is similar as for $\preceq$, with the restriction that all vertex sets are the same. Let $H_{i}=\left(V, E_{i}\right), i \in I$, any family of hypergraphs having the same vertex set. As for $\preceq$, it is easy to show that $\wedge_{i \in I} H_{i}=\left(V, \cap_{i \in I} E_{i}\right)$ and $\vee_{i \in I} H_{i}=\left(V, \cup_{i \in I} E_{i}\right)$. The smallest element is $(V, \emptyset)$ and the largest element is $(V, \mathcal{E})$.

This is simply a restriction of $\preceq$ by considering only the hypergraphs with the same set of vertices, so it will not be further considered (once $V$ is fixed, it would be equivalent to consider only $(\mathcal{P}(\mathcal{E}), \subseteq))$.

\subsection{Partial ordering based on the notion of sub-hypergraph and induced sub-hypergraph}

In this section, we provide a few other examples of possible partial orderings on hypergraphs, based on the natural orderings induced by the definitions of sub-hypergraph and induced subhypergraph.

Let us first consider the sub-hypergraphs and the derived natural partial ordering.

Definition 3. $H_{1} \preceq{ }_{s} H_{2}$ if $H_{1}$ is a sub-hypergraph of $H_{2}$, i.e.:

$$
\forall\left(H_{1}, H_{2}\right) \in \mathcal{T}^{2}, H_{1} \preceq_{s} H_{2} \Longleftrightarrow\left\{\begin{array}{l}
V_{1} \subseteq V_{2} \\
E_{1}=\left\{e \in E_{2} \mid v(e) \subseteq V_{1}\right\}
\end{array}\right.
$$

Note that the condition on the hyperedges is stronger than $E_{1} \subseteq E_{2}$ since $E_{1}$ should contain all hyperedges of $E_{2}$ composed of vertices of $V_{1}$

Another possibility would be to define a partial ordering $\preceq_{s}^{\prime}$ by replacing the equality in the condition on the hyperedges by an inclusion:

\section{Definition 4}

$\forall\left(H_{1}, H_{2}\right) \in \mathcal{T}^{2}, H_{1} \preceq_{s}^{\prime} H_{2} \Longleftrightarrow\left\{\begin{array}{l}V_{1} \subseteq V_{2} \\ E_{1} \subseteq\left\{e \in E_{2} \mid v(e) \subseteq V_{1}\right\}\end{array}\right.$

Proposition 4. $\preceq_{s}$ and $\preceq_{s}^{\prime}$ are partial orderings on $\mathcal{T}$.

Proof. Let $H_{1}=\left(V_{1}, E_{1}\right)$ and $H_{2}=\left(V_{2}, E_{2}\right)$ be any hypergraphs in $\mathcal{T}$.

Let us first take $H_{2}=H_{1}$. Then $V_{1}=V_{2}$, and $E_{1}=\left\{e \in E_{1}\right.$ $\left.\mid v(e) \subseteq V_{1}\right\}$. Hence $\preceq_{s}$ is reflexive. If $H_{1}{ }_{s} H_{2}$ and $H_{2}{ }_{s} H_{1}$, then $V_{1}=V_{2}$. We also have $E_{1}=\left\{e \in E_{2} \mid v(e) \subseteq V_{1}\right\}=\left\{e \in E_{2} \mid v(e) \subseteq V_{2}\right\}=$ $E_{2}$, and thus $\preceq_{s}$ is anti-symmetric. If $H_{1}{ }_{s} H_{2}$ and $H_{2} \preceq_{s} H_{3}$, then $V_{1} \subseteq V_{2} \subseteq V_{3}$. For hyperedges, we have $E_{1}=\left\{e \in E_{2} \mid v(e) \subseteq V_{1}\right\}=$ $\left\{e \in E_{3} \mid v(e) \subseteq V_{1}\right.$ and $\left.v(e) \subseteq V_{2}\right\}=\left\{e \in E_{3} \mid v(e) \subseteq V_{1} \cap V 2=V 1\right\}$, which shows that $\preceq_{s}$ is transitive. Hence it is a partial ordering.

Let us now consider $\preceq_{s}^{\prime}$. The reasoning for the vertex sets is the same as for $\preceq_{s}$. If $H_{2}=H_{1}$, then $\left\{e \in E_{1} \mid \mathcal{u}(e) \subseteq V_{1}\right\}=E_{1}$, and $\preceq_{s}^{\prime}$ is reflexive. If $H_{1} \preceq_{s}^{\prime} H_{2}$ and $H_{2} \preceq_{s}^{\prime} H_{1}, E_{1} \subseteq\left\{e \in E_{2} \mid v(e) \subseteq V_{1}=V_{2}\right\}=E_{2}$. Similarly $E_{2} \subseteq E_{1}$ and $E_{1}=E_{2}$. Hence $\preceq_{s}^{\prime}$ is anti-symmetric. If $H_{1} \swarrow_{s}^{\prime} H_{2}$ and $H_{2} \swarrow_{s}^{\prime} H_{3}$, then $E_{1} \subseteq\left\{e \in E_{2} \mid v(e) \subseteq V_{1}\right\} \subseteq\left\{e \in E_{3}\right.$ $\mid v(e) \subseteq V_{1}$ and $\left.v(e) \subseteq V_{2}\right\}=\left\{e \in E_{3} \mid v(e) \subseteq V_{1} \cap V_{2}=V_{1}\right\}$. Hence $\preceq_{s}^{\prime}$ is transitive and it is a partial ordering.
Definition 5. A partial ordering can be defined from the type of inclusion which is implicit in the definition of induced sub-hypergraph, as:

$\forall\left(H_{1}, H_{2}\right) \in \mathcal{T}^{2}, H_{1} \preceq_{i} H_{2} \Longleftrightarrow\left\{\begin{array}{l}V_{1} \subseteq V_{2} \\ E_{1}=\left\{\left\{v(e) \cap V_{1}\right\} \mid e \in E_{2}\right\}\end{array}\right.$

i.e. $H_{1}$ is the sub-hypergraph induced by $H_{2}$ for $V_{1}$.

Proposition 5. The relation $\preceq_{i}$ is a partial ordering on $\mathcal{T}$.

The proof is similar to the one for $\preceq_{s}$.

It might be more suitable (to allow for more frequent comparisons between hypergraphs) to propose a less strict version where $E_{1}$ is only required to be included in the set of hyperedges of the induced sub-hypergraph (as for $\preceq_{s}^{\prime}$ ):

\section{Definition 6.}

$\forall\left(H_{1}, H_{2}\right) \in \mathcal{T}^{2}, H_{1} \preceq_{i}^{\prime} H_{2} \Longleftrightarrow\left\{\begin{array}{l}V_{1} \subseteq V_{2} \\ E_{1} \subseteq\left\{\left\{v(e) \cap V_{1}\right\} \mid e \in E_{2}\right\}\end{array}\right.$

Proposition 6. The following properties hold:

- $\preceq_{i}^{\prime}$ is a partial ordering on $\mathcal{T}$.

- $\left(\mathcal{T}, \preceq_{i}^{\prime}\right)$ is a complete lattice.

- The infimum is: $H_{1} \wedge_{i}^{\prime} H_{2}=\left(V_{1} \cap V_{2},\left\{e^{\prime}=\left\{v(e) \cap V_{1} \cap V_{2}\right\}\right.\right.$ $\left.\left.\mid e \in E_{1} \cap E_{2}\right\} \cap \mathcal{E}\right)$, and its extension to any family $\left(H_{j)_{j} \in J}\right.$ : $\wedge_{i}^{\prime}\left(H_{j}\right)_{j \in J}=\left(\cap_{j \in J} V_{j},\left\{e^{\prime}=\left\{v(e) \cap\left(\cap_{j \in J} V_{j}\right)\right\} \mid e \in \cap_{j \in J} E_{j}\right\} \cap \mathcal{E}\right)$.

- The supremum is: $H_{1} \vee_{i}^{\prime} H_{2}=\left(V_{1} \cup V_{2}, E_{1} \cup E_{2}\right)$, and its extension to any family $\left(H_{i}\right)$.

- The smallest element is $H_{\emptyset}=(\emptyset, \emptyset)$ and the largest element is $\mathcal{H}=(\mathcal{V}, \mathcal{E})$.

Proof. It is easy to show that $\preceq_{i}^{\prime}$ is a partial ordering (the proof is similar as the one for $\preceq_{s}^{\prime}$ ).

Let $H_{1}=\left(V_{1}, E_{1}\right)$ and $H_{2}=\left(V_{2}, E_{2}\right)$ be any hypergraphs in $\mathcal{T}$, and let $H=\left(V_{1} \cup V_{2}, E_{1} \cup E_{2}\right)$. We have $V_{1} \subseteq V_{1} \cup V_{2}$ and $V_{2} \subseteq V_{1} \cup V_{2}$. Let $e \in E_{1}$. We have $v(e) \cap V_{1}=v(e), e \in E_{1} \cup E_{2}$, and therefore $e \in\left\{\left\{u(e) \cap V_{1}\right\} \mid e \in E_{1} \cup E_{2}\right\}$. Similarly $E_{2} \subseteq\left\{\left\{u(e) \cap V_{2}\right\} \mid e \in E_{1} \cup E_{2}\right\}$. Hence $H_{1} \preceq_{i}^{\prime} H$ and $H_{2} \preceq_{i}^{\prime} H$. Let us now assume that $H_{1} \preceq_{i}^{\prime} H_{3}$ and $H_{2} \preceq_{i}^{\prime} H_{3}$. We have $V_{1} \subseteq V_{3}$ and $V_{2} \subseteq V_{3}$, hence $V_{1} \cup V_{2} \subseteq V_{3}$. We also have $E_{1} \cup E_{2} \subseteq\left\{\left\{v(e) \cap V_{1}\right\} \mid e \in E_{3}\right\} \cup\left\{\left\{v(e) \cap V_{2}\right\} \mid e \in E_{3}\right\}$ $\subseteq\left\{\left\{u(e) \cap\left(V_{1} \cup V_{2}\right)\right\} \mid e \in E_{3}\right\}$. Hence $H \preceq_{i}^{\prime} H_{3}$. Finally $H$ is the least upper bound and $H_{1} \vee_{i}^{\prime} H_{2}=\left(V_{1} \cup V_{2}, E_{1} \cup E_{2}\right)$. This reasoning extends directly to any family of hypergraphs $\left(H_{i}\right)$.

Now let $\quad V=V_{1} \cap V_{2}, E=\left\{\left\{v(e) \cap V_{1} \cap V_{2}\right\} \mid e \in E_{1} \cap E_{2}\right\} \cap \mathcal{E}$ and $H=(V, E)$. We have $V \subseteq V_{1}$ and $V \subseteq V_{2}$. Let $e \in E$. Then $\exists e^{\prime} \in E_{1} \cap E_{2}$ such that $\chi(e)=v\left(e^{\prime}\right) \cap V_{1} \cap V_{2}$. We also have $v(e)=v\left(e^{\prime}\right) \cap V_{1}=v\left(e^{\prime}\right) \cap V_{2}$. Since $e^{\prime} \in E_{1}$, it belongs to the set $\left\{\left\{u\left(e^{\prime}\right) \cap V_{2}\right\} \mid e^{\prime} \in E_{1}\right\}$. Similarly, $e^{\prime} \in E_{2}$ and thus it belongs to the set $\left\{\left\{v\left(e^{\prime}\right) \cap V_{1}\right\} \mid e^{\prime} \in E_{2}\right\}$. Hence we have $H \preceq_{i}^{\prime} H_{1}$ and $H \preceq_{i}^{\prime} H_{2}$. Let us now consider any hypergraph $H_{3}=\left(V_{3}, E_{3}\right)$ such that $H_{3} \preceq_{1}^{\prime} H_{1}$ and $H_{3} \preceq_{i}^{\prime} H_{2}$. We have $V_{3} \subseteq V_{1}$ and $V_{3} \subseteq V_{2}$, thus $V_{3} \subseteq V$. Since $E_{3} \subseteq\left\{\left\{u(e) \cap V_{3}\right\} \mid e \in E_{1}\right\}$ and $E_{3} \subseteq\left\{\left\{u(e) \cap V_{3}\right\} \mid e \in E_{2}\right\}$, we have $E_{3} \subseteq\left\{\left\{u(e) \cap V_{1} \cap V_{2}\right\} \mid e \in E_{1} \cap E_{2}\right\}$, and $H_{3} \swarrow_{i}^{\prime} H$. Hence $H$ is the largest lower bound and $H=H_{1} \wedge_{i}^{\prime} H_{2}$. The proof for any family is similar.

This shows that $\left(\mathcal{T}, \preceq_{i}^{\prime}\right)$ is a complete lattice. The smallest element is $\Lambda \mathcal{T}=\left(\cap_{V \in \mathcal{V}} V,\left\{\left\{v(e) \cap\left(\cap_{V \in \mathcal{V}} V\right)\right\} \mid e \in \cap_{E \in \mathcal{E}} E\right\} \cap \mathcal{E}\right)=(\emptyset, \emptyset)=H_{\emptyset}$. The largest element is $\bigvee \mathcal{T}=\left(\cup_{V \in \mathcal{V}} V, \cup_{E \in \mathcal{E}} E\right)=(\mathcal{V}, \mathcal{E})=\mathcal{H}$.

Another idea involves isomorphisms, as in the following definition. 
Definition 7. Let $\bar{H}$ be the set of isomorphism classes of hypergraphs. A partial order on $\bar{H}$ can be defined, for all $H_{1}, H_{2}$ in $\bar{H}$ as:

$H_{1} \leqslant f H_{2} \Longleftrightarrow H_{1}$ is isomorphic (by $f$ ) to an induced subhypergraph of $H_{2}$

Proposition 7. The structure $\left(\bar{H}, \leqslant_{f}\right)$ is a complete lattice. The supremum is $\sup \left\{H_{1}, H_{2}\right\}=H_{1} \vee H_{2}$ (as in Proposition 2 for $\preceq$ ), and the infimum $\inf \left\{H_{1}, H_{2}\right\}$ is the maximum common induced subhypergraph (and their extension to any family).

Proof. Let us provide a sketch of the proof. It is easy to show that $\leqslant_{f}$ defines a partial ordering on $\bar{H}$. The reflexivity and the anti-symmetry follow from the fact that an hypergraph without repeated hyperedge is its own induced subhypergraph. The proof for the supremum is similar to the one for the other partial orderings considered before. For the infimum, it is the largest lower bound by construction.

\subsection{Discussion}

The partial ordering $\preceq$ and the corresponding lattice have clear advantages over the simple examples $(\mathcal{P}(\mathcal{V}), \subseteq)$ and $(\mathcal{P}(\mathcal{E}), \subseteq)$ mentioned at the beginning of this section, since both an ordering on vertices and an ordering on hyperedges are considered, thus taking the hypergraph structure into account. As already mentioned, $\preceq_{p}$ is the same as $\preceq$ when ordering hypergraphs having the same sets of vertices, which does not deserve to be further investigated.

The other proposed partial orderings $\preceq_{s}$ and $\preceq_{i}$ (or $\preceq_{s}^{\prime}$ and $\preceq_{i}^{\prime}$ ) have the additional feature of imposing stronger links between the structures of the two hypergraphs to be ordered than $\preceq$. These partial orderings may be interesting when the notions of (induced) sub-hypergraphs are explicitly involved in the application at hand. Examples include all problems where sub-hypergraphs isomorphisms have to be considered, for instance if partial views of a scene, each of which being represented by a sub-hypergraph, have to be compared, or if a matching between a partial view and the complete scene has to be found.

An advantage of $\preceq$ is that supremum and infimum are very easy to compute. While they can also be computed quite easily for $\preceq_{i}^{\prime}$, this is not straightforward for $\preceq_{i}$ and $\preceq_{s}$, and close forms could not be directly obtained.

In the following, we use $\preceq$ for defining in a general way a partial ordering between two hypergraphs.

\section{Mathematical morphology on hypergraphs}

\subsection{Algebraic dilation and erosion}

Once we have a complete lattice, the whole algebraic apparatus of mathematical morphology applies.

Let $\left(\mathcal{T}, \preceq\right.$ ) and $\left(\mathcal{T}^{\prime}, \preceq^{\prime}\right)$ be two complete lattices (which can be any of those defined in Section 3, and do not need to be equal). All the following definitions and results are common to the general algebraic framework of mathematical morphology in complete lattices $[2,5,6,8,25]$.

Definition 8. An operator $\delta: \mathcal{T} \rightarrow \mathcal{T}^{\prime}$ is a dilation if: $\forall\left(x_{i}\right) \in \mathcal{T}, \delta\left(\vee_{i} x_{i}\right)=\vee_{i}^{\prime} \delta\left(x_{i}\right)$, where $\vee$ denotes the supremum associated with $\preceq$ and $\vee^{\prime}$ the one associated with $\preceq^{\prime}$. An operator $\varepsilon: \mathcal{T}^{\prime} \rightarrow \mathcal{T}$ is an erosion if: $\forall\left(x_{i}\right) \in \mathcal{T}^{\prime}, \varepsilon\left(\wedge_{i}^{\prime} x_{i}\right)=\wedge_{i} \varepsilon\left(x_{i}\right)$, where $\wedge$ and $\wedge^{\prime}$ denote the infimum associated with $\preceq$ and $\preceq^{\prime}$, respectively.

All classical properties of mathematical morphology then hold $[5,6,25]$, and are therefore not recalled here.
An important notion is the one of adjunction. A pair or operators $(\varepsilon, \delta)$, with $\varepsilon:\left(\mathcal{T}^{\prime}, \preceq^{\prime}\right) \rightarrow(\mathcal{T}, \preceq)$ and $\delta:(\mathcal{T}, \preceq) \rightarrow\left(\mathcal{T}^{\prime}, \preceq^{\prime}\right)$ is an adjunction if and only if $\forall X \in \mathcal{T}, \forall Y \in \mathcal{T}^{\prime}, X \preceq \varepsilon(Y) \Longleftrightarrow \delta(X) \preceq^{\prime} Y$. A classical result is that if $(\varepsilon, \delta)$ form an adjunction, then $\varepsilon$ is an erosion and $\delta$ is a dilation. The compositions $\delta \varepsilon$ and $\varepsilon \delta$ are then an opening (increasing, idempotent and anti-extensive operator) and a closing (increasing, idempotent and extensive operator), respectively.

\subsection{Structuring element and morphological operations}

In classical morphology dilations and erosions can be expressed by means of a set, called structuring element, which defines a neighborhood at each point [1], and this idea has been used for graphs as well [20]. The structuring element "centered" at $x$ is denoted by $B_{x}=\delta(\{x\})$. More generally, the structuring element can be interpreted as a binary relation between two elements, thus enabling the extension of this idea to any lattice.

Defining morphological dilations on hypergraphs calls for canonical decompositions of the elements of the considered lattice.

In the case of the lattice $(\mathcal{P}(\mathcal{V}), \subseteq)$, each subset of vertices $V$ can be trivially decomposed as $V=\cup_{x \in V}\{x\}$, and a morphological dilation then writes $\delta_{B}(V)=\cup_{x \in V} B_{x}=\cup_{x \in V} \delta(\{x\})$.

In the case of the lattice $(\mathcal{P}(\mathcal{E}), \subseteq)$, each subset of hyperedges $E$ can be decomposed as $E=\cup_{e \in E}\{e\}$, and a morphological dilation is then $\delta_{B}(E)=\cup_{e \in E} B_{e}=\cup_{e \in E} \delta(\{e\})$.

Let us now consider the lattice of hypergraphs, with the partial ordering $\preceq$ (see Definition 1 ). Let $H=(V, E)$ be a hypergraph of this lattice. For $E$, a natural decomposition consists of $E=\cup_{e \in E}\{e\}$. For $V$ the decomposition should be consistent with the one of $E$, in order to associate an "elementary" hypergraph to each $e$. We thus consider $v(e)$, the set of vertices associated with $e$. Additionally, the decomposition should also involve all vertices that do not belong to any hyperedge. We denote by $V_{\backslash E}$ this set of vertices. Finally we propose the following canonical decomposition of $H$, from its sup generating property: $H=\left(\vee_{e \in E}(v(e),\{e\})\right) \vee$ $\left(\vee_{x \in V_{\backslash E}}(\{x\}, \emptyset)\right)$.

The question of how the structuring element should be defined depends on the application and on the type of desired results. Examples are provided next.

\subsection{Examples}

Example 1. Let us consider $\mathcal{T}=(\mathcal{P}(\mathcal{E}), \subseteq)$. An example of structuring element, defining the elementary dilation of each hyperedge, consists in taking all hyperedges which have at least one vertex in common with the considered hyperedge:

$\forall e \in E, B_{e}=\delta(\{e\})=\left\{e^{\prime} \in \mathcal{E} \mid v(e) \cap v\left(e^{\prime}\right) \neq \emptyset\right\}$

where the intersection applies on the sets of vertices defining $e$ and $e^{\prime}$. Dilating a subset $E$ by this structuring element means adding all hyperedges that are directly connected to $E$.

As an illustration, let us consider the two hypergraphs depicted in Fig. 1. For the first one, we have for instance $\delta\left(\left\{e_{1}\right\}\right)=\delta\left(\left\{e_{3}\right\}\right)$ $=\left\{e_{1}, e_{2}, e_{3}, e_{4}\right\}, \delta\left(\left\{e_{2}\right\}\right)=\left\{e_{1}, e_{2}, e_{3}\right\}, \delta\left(\left\{e_{4}\right\}\right)=\left\{e_{1}, e_{3}, e_{4}\right\}$, and for the second one, $\delta\left(\left\{e_{i}\right\}\right)=\left\{e_{1}, e_{2}, e_{2}\right\}$, for $i=1,2,3$.

Let us now consider the adjoint erosion. It is defined by $\forall E \in \mathcal{P}(\mathcal{E}), \varepsilon(E)=\cup\left\{E^{\prime} \in \mathcal{P}(\mathcal{E}) \mid \delta\left(E^{\prime}\right) \subseteq E\right\}=\cup\left\{E^{\prime} \in \mathcal{P}(\mathcal{E}) \mid \cup_{e^{\prime} \in E^{\prime}} \delta\left(e^{\prime}\right) \subseteq E\right\}=$ $\cup\left\{E^{\prime} \in \mathcal{P}(\mathcal{E}) \mid \forall e^{\prime} \in E^{\prime}, \delta\left(e^{\prime}\right) \subseteq E\right\}$. In the second example in Fig. 1 (right), we have $\varepsilon\left(\left\{e_{1}, e_{2}, e_{3}\right\}\right)=\left\{e_{1}, e_{2}, e_{3}\right\}, \varepsilon\left(\left\{e_{1}, e_{2}\right\}\right)=\emptyset$, etc. (the erosions of all subsets of $\left\{e_{1}, e_{2}, e_{3}\right\}$ are empty, except for $\left.\left\{e_{1}, e_{2}, e_{3}\right\}\right)$. 

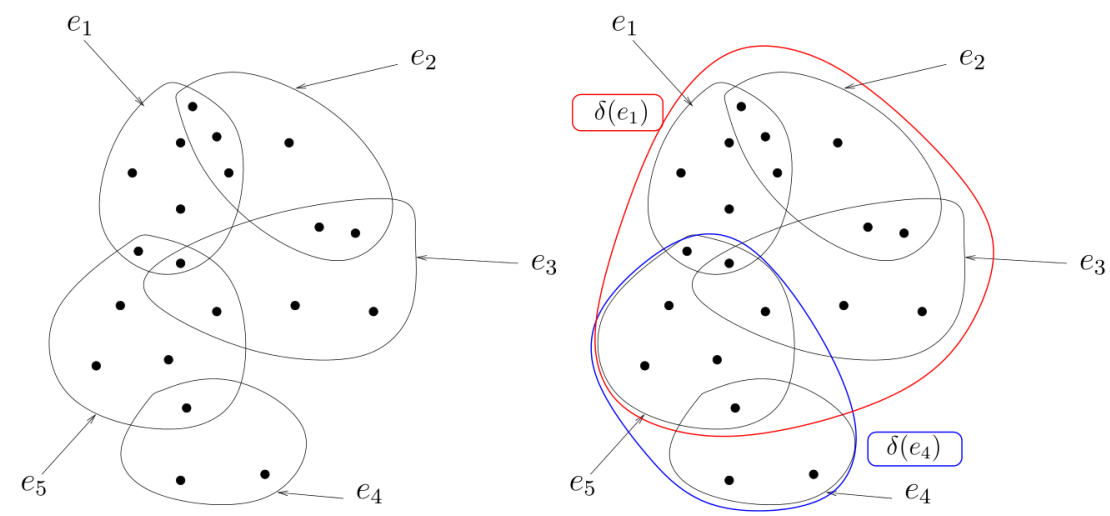

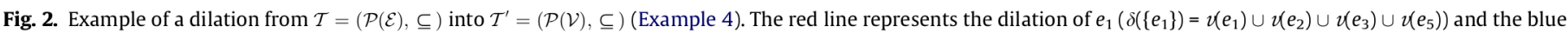
line the dilation of $e_{4}\left(\delta\left(\left\{e_{4}\right\}\right)=\imath\left(e_{4}\right) \cup v\left(e_{5}\right)\right)$. (For interpretation of the references to color in this figure legend, the reader is referred to the web version of this article.)
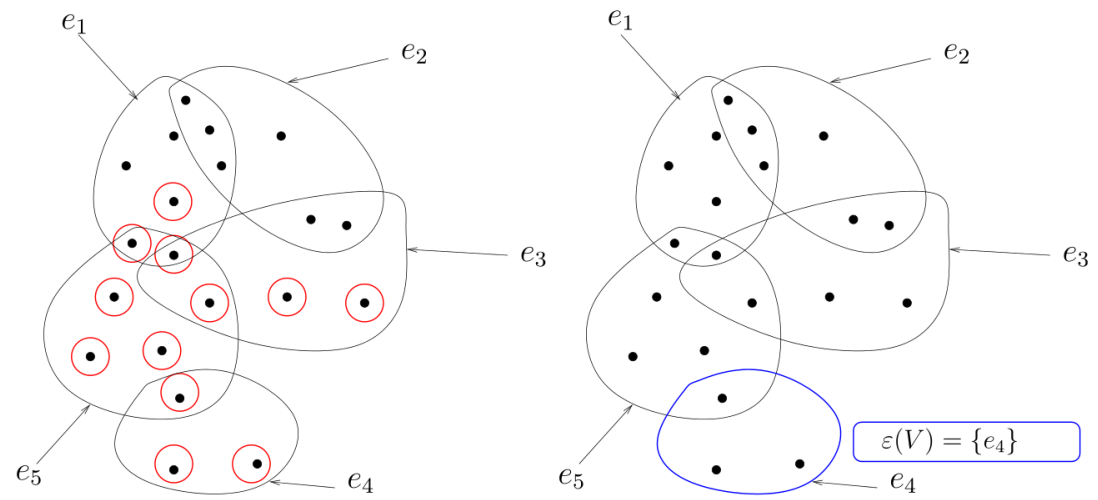

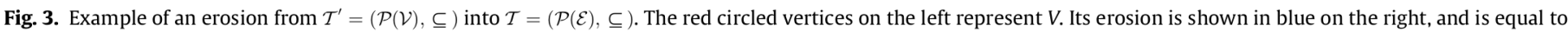
$\left\{e_{4}\right\}$. (For interpretation of the references to color in this figure legend, the reader is referred to the web version of this article.)

Example 2. Another example, where less hyperedges are added, can be obtained by imposing a minimal cardinality on the intersection: $\forall e \in E, B_{e}^{k}=\left\{e^{\prime} \in \mathcal{E}|| v(e) \cap v\left(e^{\prime}\right) \mid \geqslant k\right\}$. Note that such a constraint could not be imposed on graphs for $k>2$, which is an example of the enhanced features provided by hypergraph representations. Let us consider again the example in Fig. 1 left. For $k=2$, we obtain now $\delta\left(\left\{e_{1}\right\}\right)=\left\{e_{1}, e_{3}, e_{4}\right\}, \delta\left(\left\{e_{2}\right\}\right)=\left\{e_{2}\right\}, \quad \delta\left(\left\{e_{3}\right\}\right)$ $=\delta\left(\left\{e_{4}\right\}\right)=\left\{e_{1}, e_{3}, e_{4}\right\}$. For $k=3$, the dilations of $e_{1}$ and $e_{3}$ are even less extended: $\delta\left(\left\{e_{1}\right\}\right)=\left\{e_{1}, e_{4}\right\}$ and $\delta\left(\left\{e_{3}\right\}\right)=\left\{e_{3}, e_{4}\right\}$.

Example 3. Let us now consider dilations from $\mathcal{T}=(\mathcal{P}(\mathcal{E}), \subseteq)$ into $\mathcal{T}^{\prime}=(\mathcal{P}(\mathcal{V}), \subseteq)$. This will be useful later on when considering dual hypergraphs (see Section 5 ). Then the elementary dilation should map a hyperedge to a subset of vertices. A trivial example is: $\forall e \in E, B_{e}=\delta(\{e\})=\{x \in \mathcal{V} \mid x \in v(e)\}=v(e)$. This achieves the required mapping, but it is not very relevant and interesting from a practical point of view, since no additional vertex is added by the dilation of a hyperedge. In a similar way, we can define in a straightforward way a dilation $\delta$ from $(\mathcal{P}(V), \subseteq)$ into $(\mathcal{P}(E), \subseteq)$, as: $\forall x \in V, \delta(\{x\})=\{e \in E \mid x \in \mathcal{V}(e)\}$, and $\forall X \subseteq V, \delta(X)=\cup_{x \in X} \delta(\{x\})$.

Example 4. More interestingly, we can define a structuring element as in Example 1, but considering the resulting subset of vertices to define a dilation from $\mathcal{T}=(\mathcal{P}(\mathcal{E}), \subseteq)$ into $\mathcal{T}^{\prime}=(\mathcal{P}(\mathcal{V}), \subseteq)$ : $\forall e \in E, B_{e}=\delta(\{e\})=\left\{x \in \mathcal{V} \mid \exists e^{\prime} \in \mathcal{E}, x \in \mathcal{V}\left(e^{\prime}\right)\right.$ and $\left.v(e) \cap v\left(e^{\prime}\right) \neq \emptyset\right\}$ $=\cup\left\{v\left(e^{\prime}\right) \mid v\left(e^{\prime}\right) \cap v(e) \neq \emptyset\right\}$. An example is illustrated in Fig. 2. As in Example 2, we could add more strict constraints on the intersection, if we want the dilation to include less vertices.
Let us consider the adjoint erosion $\varepsilon$, from $\mathcal{T}^{\prime}$ into $\mathcal{T}$. It is given by:

$$
\begin{aligned}
\forall V & \in \mathcal{P}(\mathcal{V}), \quad \varepsilon(V)=\cup\{E \in \mathcal{P}(\mathcal{E}) \mid \forall e \in E, \delta(\{e\}) \subseteq V\}=\left\{e \in \mathcal{E} \mid \forall e^{\prime}\right. \\
& \left.\in \mathcal{E}, \quad v\left(e^{\prime}\right) \cap v(e) \neq \emptyset \Rightarrow v\left(e^{\prime}\right) \subseteq V\right\} .
\end{aligned}
$$

Let us consider the example in Fig. 2, and $V$ as illustrated in Fig. 3. We have $\varepsilon(V)=\left\{e_{4}\right\}$. An opening can be defined from an adjunction as $\delta \varepsilon$. In this example, the opening of $V$ is $\delta \varepsilon(V)=\delta\left(\left\{e_{4}\right\}\right)=$ $v\left(e_{4}\right) \cup v\left(e_{5}\right)$ and it is the set of all vertices enclosed in the blue line in Fig. 2. This simple example illustrates the filtering effect of the opening, which removes all vertices of $V$ that do not belong to hyperedges $e$ such that $v(e) \subseteq V$, as illustrated in Fig. 4. As for closing we have for this example $\varepsilon \delta\left(\left\{e_{4}\right\}\right)=\left\{e_{4}\right\}$ and $\varepsilon \delta\left(\left\{e_{1}\right\}\right)=$ $\varepsilon\left(\cup_{i=1,2,3,5} v\left(e_{i}\right)\right)=\left\{e_{1}, e_{2}, e_{3}\right\}$.

Example 5. Let us now consider $\mathcal{T}=(\{H=(V, E)\}, \preceq)$. An elementary dilation can be defined according to the proposed canonical decomposition as: $\forall x \in V_{\backslash E}, \delta(\{x\}, \emptyset)=(\{x\}, \emptyset)$, for isolated vertices, and for elementary hypergraphs associated with hyperedges: $\forall e \in E, \delta(v(e),\{e\})=\left(\cup\left\{v\left(e^{\prime}\right) \mid v\left(e^{\prime}\right) \cap v(e) \neq \emptyset\right\},\left\{e^{\prime} \in \mathcal{E} \mid v\left(e^{\prime}\right) \cap v(e)\right.\right.$ $\neq \emptyset\}$ ). As an illustration, let us consider the example in Fig. 2. Let $\mathcal{V}=\cup_{i=1 \ldots .5} v\left(e_{i}\right)$ (i.e. all vertices shown in the figure), $\mathcal{E}=\left\{e_{1}, \ldots, e_{5}\right\}$, and $\mathcal{H}=(\mathcal{V}, \mathcal{E})$. In the lattice $\mathcal{T}$ defined on $\mathcal{H}$, let us take $H=(V, E)$, with $V=v\left(e_{2}\right) \cup v\left(e_{3}\right)$ and $E=\left\{e_{2}, e_{3}\right\}$. The canonical decomposition of $H$ is: $H=\left(v\left(e_{2}\right),\left\{e_{2}\right\}\right) \vee\left(v\left(e_{3}\right),\left\{e_{3}\right\}\right)$. Its dilation is then $\delta(H)=\delta\left(v\left(e_{2}\right),\left\{e_{2}\right\}\right) \vee \delta\left(v\left(e_{3}\right),\left\{e_{3}\right\}\right)=\left(v\left(e_{1}\right) \cup v\left(e_{2}\right) \cup v\left(e_{3}\right)\right.$, $\left.\left\{e_{1}, e_{2}, e_{3}\right\}\right) \vee\left(u\left(e_{1}\right) \cup v\left(e_{2}\right) \cup v\left(e_{3}\right) \cup v\left(e_{5}\right),\left\{e_{1}, e_{2}, e_{3}, e_{5}\right\}\right)=\left(\cup_{i=1,2,3,5} v\left(e_{i}\right)\right.$, $\left.\left\{e_{1}, e_{2}, e_{3}, e_{5}\right\}\right)$. This is illustrated in Fig. 5. 

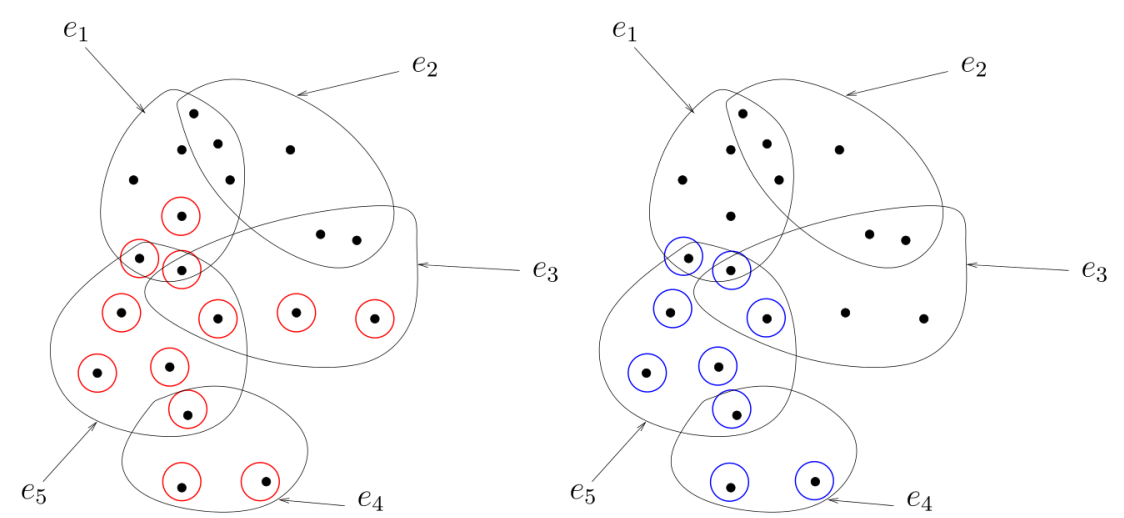

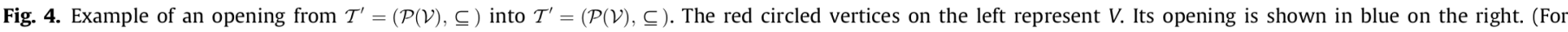
interpretation of the references to color in this figure legend, the reader is referred to the web version of this article.)
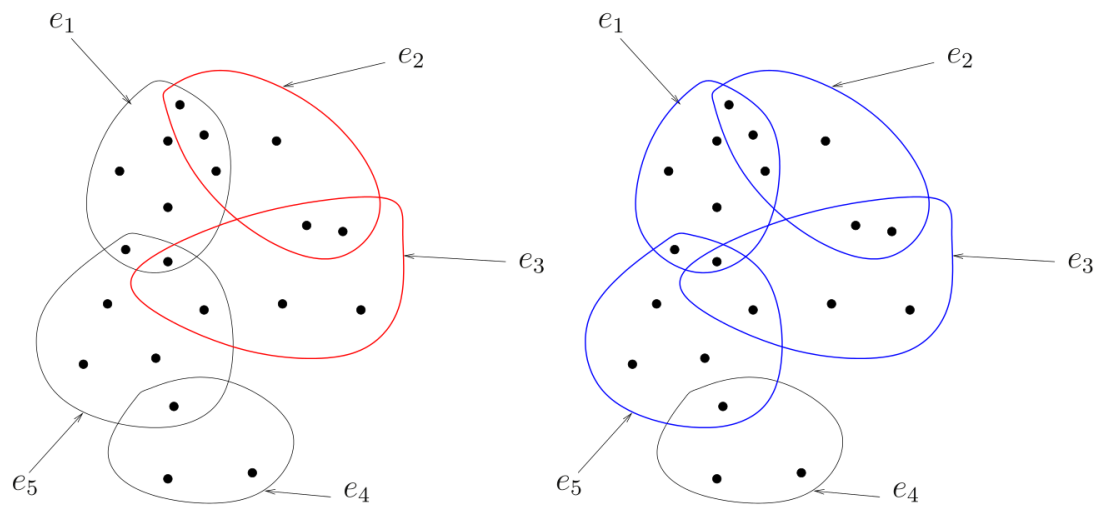

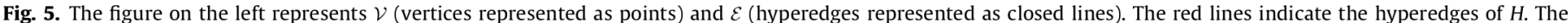

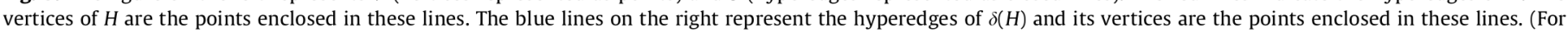
interpretation of the references to color in this figure legend, the reader is referred to the web version of this article.)

Note that if we consider also attributes on the vertices (or hyperedges), other examples can be provided by using a similarity between attributes. For instance isolated vertices could be dilated by adding all vertices that have similar attribute values.

Example 6. In our last example, we show how a specific dilation is linked to the notion of transversal of a hypergraph. A transversal (or hitting set) of a hypergraph $H=(V, E)$ is a set $T \subseteq V$ that has non-empty intersection with every hyperedge, i.e. for all $e \in E$, $T \cap v(e) \neq \emptyset$. Computing the transversal of a hypergraph has applications in machine learning, game theory, indexing of databases, SAT problems, data mining, optimization, etc. (see [26]). Let us define:

$\forall V^{\prime} \subseteq V, E\left(V^{\prime}\right)=\left\{e \in E \mid \exists x \in V^{\prime}, x \in v(e)\right\}$,

i.e. the set of the hyperedges that hit $V^{\prime}$, and

$\forall E^{\prime} \subseteq E, V\left(E^{\prime}\right)=\left\{x \in V \mid \exists e \in E^{\prime}, x \in v(e)\right\}$,

i.e. the set of vertices of all hyperedges of $E^{\prime}$, which can be equivalently written as $V\left(E^{\prime}\right)=\cup_{e \in E^{\prime}} v(e)$. Let us consider the two lattices $(\mathcal{P}(V), \subseteq)$ and $(\mathcal{P}(E), \supseteq)$ (i.e. the partial ordering is reversed in the second one). Supremum and infimum are $\cup$ and $\cap$ for the first one, and $\cap$ and $\cup$ for the second one. We define the two following operators:

$\delta:(\mathcal{P}(V), \subseteq) \rightarrow(\mathcal{P}(E), \supseteq)$

$V^{\prime} \mapsto \delta\left(V^{\prime}\right)=E \backslash E\left(V^{\prime}\right)=\left\{e \in E \mid V^{\prime} \cap v(e)=\emptyset\right\}$

$\varepsilon:(\mathcal{P}(E), \supseteq) \rightarrow(\mathcal{P}(V), \subseteq)$

$E^{\prime} \mapsto \varepsilon\left(E^{\prime}\right)=V \backslash V\left(E^{\prime}\right)=V \backslash\left(\cup_{e \in E^{\prime}} v(e)\right)$
Proposition 8. The operators $\delta$ and $\varepsilon$ are a dilation and an erosion, respectively. Moreover they form an adjunction.

Proof. Let $(A, B) \in \mathcal{P}(V)^{2}$. We have: $\delta(A) \cap \delta(B)=\{e \in E \mid A \cap \vartheta(e)$ $=\emptyset\} \cap\{e \in E \mid B \cap v(e)=\emptyset\}=\{e \in E \mid A \cap \vartheta(e)=\emptyset$ and $B \cap v(e)=\emptyset\}=$ $\{e \in E \mid(A \cup B) \cap \vartheta(e)=\emptyset\}=\delta(A \cup B)$. Hence $\delta$ commutes with the supremum and is a dilation. In a similar way we have for $(A, B) \in \mathcal{P}(E)^{2} \varepsilon(A \cup B)=\varepsilon(A) \cap \varepsilon(B)$, hence $\varepsilon$ commutes with the infimum and is an erosion.

Let us now consider $A \in \mathcal{P}(V), B \in \mathcal{P}(E)$. We have $A \subseteq \varepsilon(B) \Rightarrow \delta(A) \supseteq \delta(\varepsilon(B))$ (since $\delta$ is a dilation, it is increasing). We have $\delta(\varepsilon(B))=\left\{e \in E \mid\left(V \backslash \cup_{e^{\prime} \in B} v\left(e^{\prime}\right)\right) \cap v(e)=\emptyset\right\}$. Let $e \in B$. Then $v(e) \subseteq \cup_{e^{\prime} \in B} v\left(e^{\prime}\right)$, and therefore $\left(\cup_{e^{\prime} \in B} v\left(e^{\prime}\right)\right)^{c} \cap v(e)=\emptyset$ and $e \in \delta(\varepsilon(B))$. Hence $B \subseteq \delta(\varepsilon(B))$ and $\delta(A) \supseteq B$. Conversely we show in a similar way that $\delta(A) \supseteq B \Rightarrow A \subseteq \varepsilon(B)$. Finally we have the following equivalence: $A \subseteq \varepsilon(B) \Leftrightarrow \delta(A) \supseteq B$, and therefore $(\varepsilon, \delta)$ is an adjunction.

The following result characterizes the transversal by a morphological dilation.

Theorem 1. Let $H=(V, E)$ be a hypergraph, $T \subseteq V$, and $\delta$ the dilation introduced in Eq. 10. The two following assertions are equivalent:

(i) the set $T$ is a transversal;

(ii) we have $\delta(T)=\emptyset$.

Proof. $\delta(T)=\{e \in E \mid T \cap \vartheta(e)=\emptyset\}=\emptyset \Leftrightarrow \forall e \in E, T \cap \vartheta(e) \neq \emptyset \Leftrightarrow T$ is a transversal. 

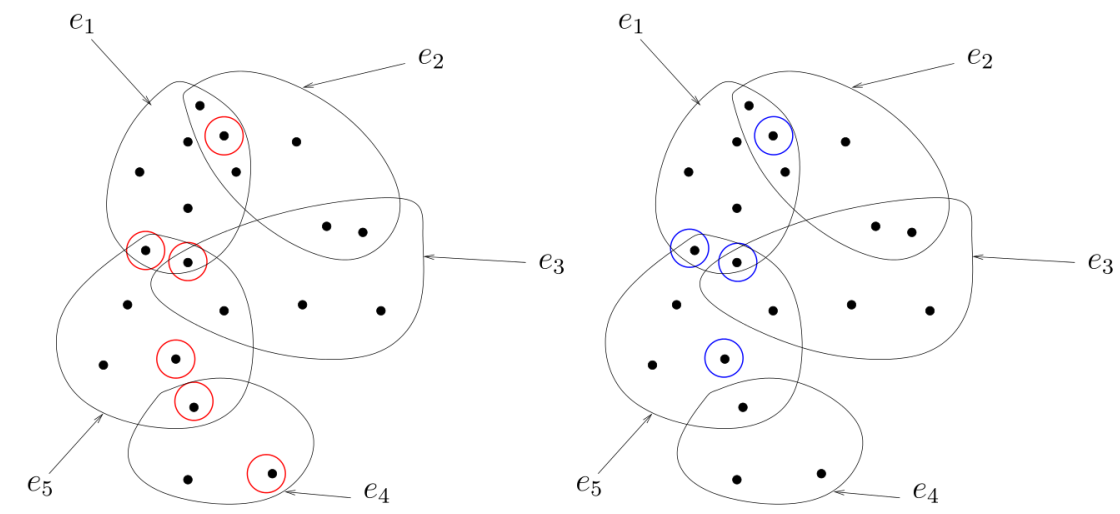

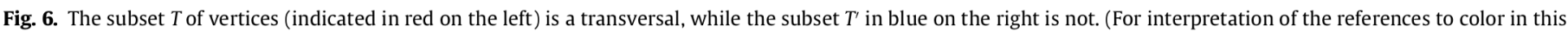
figure legend, the reader is referred to the web version of this article.)

An example is illustrated in Fig. 6. The set of vertices on the left is a transversal and its dilation is empty, while the one on the right is not (it does not intersect $e_{4}$ and its dilation is $\left\{e_{4}\right\}$, hence not empty).

\section{Dualities}

In the sequel $\delta(\{u\})$ will be simply denoted by $\delta(u)$.

Let $H=(V, E)$ be a hypergraph with $V \neq \emptyset, E \neq \emptyset$, and let $H^{*}=$ $\left(V^{*}, E^{*}\right)$ its dual. In this section we consider operators in the lattice $(\mathcal{P}(V), \subseteq)$. Let $\delta$ be such a mapping. From this mapping we define another one $\delta^{*}$, in the same lattice, such that:

$\forall x \in V, \delta^{*}(x)=\{y \in V \mid x \in \delta(y)\}$

which provides its values on singletons. We also define $\delta^{* * *}$ such that:

$\forall x \in V, \delta^{* *}(x)=\left\{y \in V \mid x \in \delta^{*}(y)\right\}$

The following proposition establishes basic results which will be useful next for deriving other results on duality, an important concept on hypergraphs. A particularly interesting result is the one expressed in Corollary 1 at the end of this section, linking morphological operators, derived rough spaces, and probability distributions.

Proposition 9. Let $H=(V, E)$ be a hypergraph with $V \neq \emptyset, E \neq \emptyset$ and $\delta$ and $\delta^{*}$ two mappings in $(\mathcal{P}(V), \subseteq)$ as introduced above; we have:

(a) for all $X \in \mathcal{P}(V), \delta^{*}(X)=\bigcup_{x \in X} \delta^{*}(x)=\{y \in V \mid X \cap \delta(y) \neq \emptyset\}$ (resp. $\delta(X)=\bigcup_{x \in X} \delta(x)=\left\{y \in V \mid X \cap \delta^{*}(y) \neq \emptyset\right\}$ ) iff $\delta^{*}$ is a dilation (resp. $\delta$ is a dilation);

(b) for all $X \in \mathcal{P}(V)$, if $\bigcup_{x \in X} \delta^{*}(x)=V$ (resp. $\bigcup_{x \in X} \delta(x)=V$ ) then $X \subseteq \bigcup_{X \cap \delta^{+}(y) \neq \emptyset} \delta^{*}(y)$ (resp. $\left.X \subseteq \bigcup_{X \cap \delta(y) \neq \emptyset} \delta(y)\right)$;

(c) $\delta^{* *}=\delta$ on V, i.e. on singletons: $\forall x \in V, \delta^{* *}(x)=\delta(x)$;

(d) if $\delta^{* *}$ and $\delta$ are dilations then $\delta^{* *}=\delta$.

\section{Proof.}

(a) Assume that $\delta^{*}$ is a dilation. The first equality is obvious by definition. Let us show the second one. Let $y \in \delta^{*}(X)$ $=\bigcup_{x \in X} \delta^{*}(x)$ then there is a $x \in X$ such that $y \in \delta^{*}(x) \Leftrightarrow x \in \delta(y)$. so $y \in\{z \in V \mid X \cap \delta(z) \neq \emptyset\}$. Let $X \in \mathcal{P}(V)$, and $y \in\{z \in V \mid$ $X \cap \delta(z) \neq \emptyset\}$, there is $x \in X$ such that $x \in \delta(y) \Leftrightarrow y \in \delta^{*}(x)$, consequently $y \in \bigcup_{x \in X} \delta^{*}(x)$.

Conversely, if the equalities hold, then it follows from the first one that $\delta^{*}$ commutes with the supremum, and is hence a dilation. (b) Obvious.

(c) Let $z \in \delta^{* *}(x)$ then $x \in \delta^{*}(z)$, and therefore $z \in \delta(x)$. In the same way $z \in \delta(x) \Rightarrow x \in \delta^{*}(z)$ and $z \in \delta^{* *}(x)$. So $\delta^{* *}=\delta$ on $V$.

(d) From the definition of a dilation.

Let $\delta:(\mathcal{P}(V), \subseteq) \rightarrow(\mathcal{P}(V), \subseteq)$ be a dilation. It gives rise to a hypergraph $H_{\delta}=\left(V,(\delta(x))_{x \in V}\right)$, where $\delta(x)$ is seen as a hyperedge built by the vertices defining $\delta(x)$.

Proposition 10. Let $\delta:(\mathcal{P}(V), \subseteq) \rightarrow(\mathcal{P}(V), \subseteq)$ be a mapping and $H=(V, E)$ be an hypergraph $(V \neq \emptyset, E \neq \emptyset)$ without isolated vertex and without repeated hyperedge. We have: $H \simeq H_{\delta} \Longleftrightarrow H^{*} \simeq H_{\delta^{*}}$.

Proof. Suppose that $H \simeq H_{\delta}$. Because a hyperedge is uniquely represented in $H$ (we assume all through this paper that hypergraphs are without repeated hyperedge), if $x \neq y$ then $\delta(x) \neq \delta(y)$, i.e. $\delta(x)=\delta(y)$ implies that $x=y$, so $\delta$ is injective on $V$.

Let $H=(V, E)$ and $H_{\delta}=\left(V_{\delta}=V, E_{\delta}=(\delta(x))_{x \in V}\right)$ be hypergraphs. We have:

$H \simeq H_{\delta} \Leftrightarrow$ there a bijection $f: V \rightarrow V_{\delta}$ such that $e \in E \Leftrightarrow f(v(e))=$ $\delta(x) \in E_{\delta}, x \in V$. Since a hyperedge of $H_{\delta}$ is uniquely represented, notice that $\left(\delta(x)_{x \in V}\right)$ is a set $\{\delta(x), x \in V\}$.

It is known that $H \simeq H_{\delta} \Longleftrightarrow H^{*} \simeq H_{\delta}^{*}$, with $H^{*}=\left(V^{*} \simeq E, E^{*} \simeq\right.$ $\left.\left(H(x)_{x \in V}\right)\right), H_{\delta}^{*}=\left(V_{\delta}^{*} \simeq(\delta(x))_{x \in V}\right), E_{\delta}^{*} \simeq\left(H(x)_{x \in V}\right)$. It is sufficient to show that $H_{\delta}^{*} \simeq H_{\delta^{*}}$, with $H_{\delta^{*}}=\left(V_{\delta^{*}} \simeq V, E_{\delta^{*}} \simeq\left(\delta^{*}(x)\right)_{\in V}\right)$. Let $g$ be a correspondence defined by:

$g:\{\delta(y), y \in V\} \rightarrow V$

$\delta(x) \mapsto g(\delta(x))=x$

Since $\delta$ is injective on $V$, we have $\delta(x)=\delta(y) \Rightarrow x=y=$ $g(\delta(x))=g(\delta(y))$, this correspondence is well defined, i.e. it is a mapping.

Clearly $g$ is surjective; moreover $g$ is injective since $|\{\delta(y), y \in V\}|=|V|$. Hence $g$ is a bijection.

Now, $H(x) \in E_{\delta}^{*} \Longleftrightarrow H(x)=\left\{\delta\left(u_{i}\right), x \in \delta\left(u_{i}\right)\right\}=\left\{\delta\left(u_{1}\right), \delta\left(u_{2}\right), \ldots\right.$, $\left.\delta\left(u_{k}\right)\right\} \in E_{\delta}^{*} \Longleftrightarrow g(H(x))=\left\{g\left(\delta\left(u_{i}\right)\right), i \in\{1,2,3, \ldots k\}\right\}=\left\{u_{1}, u_{2}\right.$, $\left.\ldots, u_{k}\right\}=\delta^{*}(x)$, because $x \in \delta\left(u_{i}\right) \Leftrightarrow u_{i} \in \delta^{*}(x)$.

Hence $H(x) \in E_{\delta}^{*} \Longleftrightarrow g(H(x))=\delta^{*}(x) \in E_{\delta^{*}}$. So $H_{\delta}^{*} \simeq H_{\delta^{x}}$, and finally $H \simeq H_{\delta} \Longleftrightarrow H^{*} \simeq H_{\delta^{*}}$.

Proposition 11. Let $H^{*}=\left(V^{*}, E^{*}\right)$ be a hypergraph and let $P=$ $\left(p_{i}\right)_{i \in\{1,2, \ldots, t\}}$ be a discrete probability distribution on $V^{*}$, taking rational values. This probability distribution gives rise to a dilation (respectively an erosion). 
Let $\delta$ be a dilation on $V^{*}$, then this dilation gives rise to a discrete probability distribution on $V^{*}$.

Proof. To prove the proposition, we will exhibit a particular dilation from $P$, and conversely a particular probability distribution from a dilation.

Let $P=\left(p_{i}\right)_{i \in\{1,2, \ldots, t\}}$ be a discrete probability distribution with rational values on $V^{*}$. For all $i \in\{1,2, \ldots, t\}$ there are $a_{i}, b_{i} \in \mathbb{N}, b_{i} \neq 0$, such that $p_{i}=\frac{a_{i}}{b_{i}}=\frac{a_{i}\left|V^{*}\right|}{b_{i}\left|V^{*}\right|}=\frac{\frac{a_{i}\left|V^{*}\right|}{b_{i}}}{\left|V^{*}\right|}$. We have:

$1=\sum_{i} p_{i}=\sum_{i} \frac{\frac{a_{i}\left|V_{i}^{*}\right|}{b_{i}}}{\left|V^{*}\right|}=\sum_{i}\left(\frac{\left\lfloor a_{i} \cdot\left|V^{*}\right|\right\rfloor}{\left|V^{*}\right|}\right)+\frac{\left|V^{*}\right|-\sum_{j}\left\lfloor\frac{a_{i}}{b_{j}} \cdot\left|V^{*}\right|\right]}{\left|V^{*}\right|}=\sum_{i}\left(\frac{\left\lfloor a_{i} \cdot\left|V^{*}\right|\right]+\left|V^{*}\right|-\sum_{j}\left[\frac{a_{i}}{b_{j}} \cdot\left|V^{*}\right|\right]}{\left|V^{*}\right|}\right)$

Let

$V_{1}^{*}=\left\{x_{1}^{*}, x_{2}^{*}, \ldots, x_{\left[\frac{a_{1}}{b_{1}}\left|V^{*}\right|\right\rfloor}^{*}\right\}$

$V_{2}^{*}=\left\{x_{\left\lfloor\frac{a_{1}}{b_{1}}\left|V^{*}\right|\right\rfloor+1}^{*}, \ldots, x_{\left\lfloor\frac{a_{1}}{b_{1}}\left|V^{*}\right|\right\rfloor+\left\lfloor\frac{a_{2}}{b_{2}},\left|V^{*}\right|\right\rfloor}^{*}\right\}, \ldots, V_{t+1}^{*}=V^{*} \backslash \bigcup_{i=1}^{t} V_{i}^{*}$

Without loss of generality, we can assume that $V_{i}^{*} \neq \emptyset$ for all $i \in\{1,2, \ldots, t+1\}$. By construction we have: $V_{i}^{*} \cap V_{j}^{*}=\emptyset$ for all $i, j \in\{1,2, \ldots, t+1\}, i \neq j$. Consequently $\left(V_{i}^{*}\right)_{i \in\{1,2, \ldots, t+1\}}$ is a partition of $V^{*}$.

The hypergraph $H^{*}=\left(V^{*}, E^{*}\right)$ can be seen as a dual of a hypergraph $H=(V, E)$. Because $V^{*} \simeq E \Longleftrightarrow \bigcup_{i=1}^{t+1} V_{i}^{*} \simeq \bigcup_{i=1}^{t+1} \bar{E}_{i}, K=$ $\left(\bar{E}_{i}\right)_{i \in\{1,2 \ldots . t+1\}}$ is a partition of $E$, where $\bar{E}_{i}$ is a subset of $E$, dual of $V_{i}^{*}$. Let us define for $A \subseteq E$

$\varepsilon(A)=\left\{\bar{E}_{i} \in K \mid \bar{E}_{i} \subseteq A\right\}$ and $\delta(A)=\left\{\bar{E}_{i} \in K \mid \bar{E}_{i} \cap A \neq \emptyset\right\}$.

It is easy to verify that $\varepsilon$ is an erosion and $\delta$ a dilation from $(\mathcal{P}(E), \subseteq)$ into $(\mathcal{P}(\mathcal{P}(E)), \subseteq)$. Note that these two operators are not directly linked to each other with an adjunction property since they are both operators from $(\mathcal{P}(E), \subseteq)$ into $(\mathcal{P}(\mathcal{P}(E)), \subseteq)$. Alternatively we can define $\varepsilon(A)=\cup\left\{\bar{E}_{i} \in K \mid \bar{E}_{i} \subseteq A\right\}$ and $\delta(A)=\cup\left\{\bar{E}_{i} \in K \mid \bar{E}_{i} \cap A \neq \emptyset\right\}$ from $(\mathcal{P}(E), \subseteq)$ into $(\mathcal{P}(E), \subseteq)$, which then form an adjunction (i.e. $\delta(A) \subseteq B \Leftrightarrow A \subseteq \varepsilon(B))$.

Now, let $\delta$ be a dilation on $V^{*}$; the relation $x_{i}^{*} \sim_{\delta} y_{j}^{*} \Longleftrightarrow \delta\left(x_{i}^{*}\right)=\delta\left(y_{j}^{*}\right)$ is an equivalence relation on $V^{*}$. We then denote by $\overline{V^{*}}{ }_{i}$ the equivalence classes:

$V^{*} / \sim_{\delta}=\left\{{\overline{V^{*}}}_{i} ; i \in\{1,2, \ldots t\}\right\}$

Let us now define $p_{i}=\frac{\left|\overline{V^{*}}\right|}{\left|\overline{V^{*}}\right|}$. We then have $0 \leqslant p_{i} \leqslant 1$ for all $i \in\{1,2, \ldots t\}$ and $\sum_{i} p_{i}=1$, thereby $\left(p_{i}\right)_{i \in\{1,2, \ldots t\}}$ is a discrete probability distribution.

This proposition is also interesting to establish links with rough sets. The definition of lower and upper approximations in terms of erosion and dilation, and the equivalence with rough sets have been developed in $[25,27]$. This result extends these notions to the case of hypergraphs, and $\varepsilon(A)$ and $\delta(A)$ exhibited in the proof are then lower and upper approximations of $A$ in a rough space (this is close to the approach proposed in [28]). Moreover, it adds a link with probabilities.

Corollary 1. Any discrete rational distribution of probability on $V^{*}$ gives rise to a rough space on $E$.

Conversely any rough space on E gives rise to a discrete rational distribution of probability on $V^{*}$.

\section{Hypergraph similarity and hypergraph kernel based on dilations}

\subsection{Hypergraph similarity}

As an example of using mathematical morphology on hypergraphs, we propose a notion of similarity between hypergraphs, based on dilations. It is well known that hypergraphs can be used to model several types of networks, such as biological, computer science, and semantic networks [29-31]. One of the most important tasks is to compare two networks. This comparison can be done using isomorphisms. However, there are two main drawbacks related to the use of isomorphisms:

- the first one concerns tractability, since there is no efficient algorithm to produce an isomorphism between two hypergraphs;

- the second one is that the isomorphism assumption is too rigid, and does not allow considering two hypergraphs as similar if they are not strictly isomorphic.

So we propose to define a new type of "comparator" between hypergraphs, based on dilation, which allows us to introduce some "tolerance" for comparing sets of hyperedges, defining a similarity as a degree of overlap between dilated sets of hyperedges.

For any hypergraph $(V, E)$, we define a dilation on the hyperedges $E$, for example as:

$$
\begin{aligned}
& \delta:(\mathcal{P}(E), \subseteq) \rightarrow(\mathcal{P}(E), \subseteq) \\
& A \mapsto \delta(A)=\{e \in E \mid v(A) \cap v(e) \neq \emptyset\}
\end{aligned}
$$

where $v(A)=\cup_{e^{\prime} \in A} v\left(e^{\prime}\right)$. In the sequel we suppose that if $\delta(A)=\emptyset$ then $A=\emptyset$ (this typically holds when $\delta$ is extensive). As before, we denote the dilation of a singleton $\{e\}$ by $\delta(e)$ to simplify notations.

Definition 9. Let $H^{1}=\left(V, E^{1}\right)$ and $H^{2}=\left(V, E^{2}\right)$ be two hypergraphs without empty hyperedge and $\delta_{E^{1}}$ and $\delta_{E^{2}}$ dilations defined on the set of hyperedges of $H^{1}$ and $H^{2}$, respectively. We define a similarity function $s$ by:

$$
\begin{aligned}
& s: \mathcal{P}\left(E^{1}\right) \times \mathcal{P}\left(E^{2}\right) \backslash(\emptyset, \emptyset) \rightarrow \mathbb{R}^{+} \\
& (A, B) \mapsto s(A, B)=\frac{\left|\delta_{E^{1}}(A) \cap \delta_{E^{2}}(B)\right|}{\left|\delta_{E^{1}}(A) \cup \delta_{E^{2}}(B)\right|}
\end{aligned}
$$

As an illustration, let us consider again the example in Fig. 1, with the definition of dilation as in Eq. (9), illustrated in Fig. 7. We have quite high similarity values, which fit with the intuition, although the hypergraphs are not isomorphic: $s\left(e_{1}, e_{i}\right)=\frac{3}{4}$, $i \in\{1,2,3\} ; \quad s\left(e_{2}, e_{i}\right)=\frac{3}{3}, i \in\{1,2,3\} ; \quad s\left(e_{3}, e_{i}\right)=\frac{3}{4}, i \in\{1,2,3\} ;$ $s\left(e_{4}, e_{i}\right)=\frac{2}{3}, i \in\{1,2,3\} ; \quad s\left(\left\{e_{1}, e_{2}\right\} ; B\right)=s\left(\left\{e_{1}, e_{3}\right\} ; B\right)=s\left(\left\{e_{1}, e_{4}\right\} ;\right.$ $B)=s\left(\left\{e_{2}, e_{3}\right\} ; B\right)=s\left(\left\{e_{2}, e_{4}\right\} ; B\right)=s\left(\left\{e_{3}, e_{4}\right\} ; B\right)=\frac{3}{4}, \quad$ for $B \subseteq E_{2}$, $B \neq \emptyset ; s\left(\left\{e_{1}, e_{2}, e_{3}\right\} ; B\right)=s\left(\left\{e_{2}, e_{3}, e_{4}\right\} ; B\right)=s\left(\left\{e_{1}, e_{3}, e_{4}\right\} ; B\right)=s\left(\left\{e_{1}\right.\right.$, $\left.\left.e_{2}, e_{4}\right\} ; B\right)=\frac{3}{4}$, for $B \subseteq E_{2}, B \neq \emptyset$; and $s\left(\left\{e_{1}, e_{2}, e_{3}, e_{4}\right\} ; B\right)=\frac{3}{4}$, for $B \subseteq E_{2}, B \neq \emptyset$.

Proposition 12. Let $H^{1}=\left(V, E^{1}\right)$ and $H^{2}=\left(V, E^{2}\right)$ be two hypergraphs without empty hyperedge, and $\delta_{E^{1}}$ and $\delta_{E^{2}}$ extensive dilations (i.e. for each hyperedge $e$, we have $\left.e \in \delta_{E^{i}}(e)\right)$ defined on $\left(\mathcal{P}\left(E^{1}\right), \subseteq\right)$ and $\left(\mathcal{P}\left(E^{2}\right), \subseteq\right)$, respectively. We have the following properties:

(a) $\forall\left(e_{i}, e_{j}\right) \in E^{1} \times E^{2}, s\left(\left(e_{i}, e_{j}\right)\right)=0 \Leftrightarrow E^{1} \cap E^{2}=\emptyset$;

(b) $\forall\left(e_{i}, e_{j}\right) \in E^{1} \times E^{2}, s\left(\left(e_{i}, e_{j}\right)\right)=1 \Rightarrow E^{1}=E^{2}$,

(c) if $E^{1}=E^{2}$ then $s$ is symmetrical. 

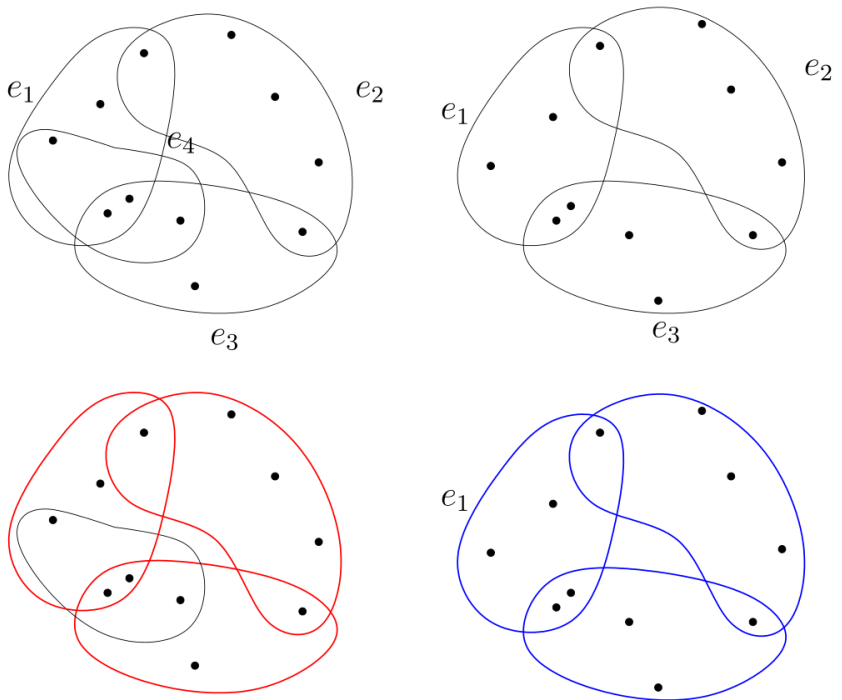

$\delta\left(e_{2}\right)$

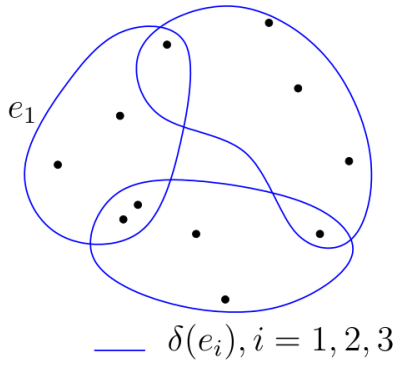

Fig. 7. Computing the similarity between two hypergraphs. Left $\left(E_{1}\right): \delta\left(e_{1}\right)=\left\{e_{1}\right.$, $\left.e_{2}, e_{3}, e_{4}\right\}, \delta\left(e_{2}\right)=\left\{e_{1}, e_{2}, e_{3}\right\}$ (shown in red on the second line left), etc. Right $\left(E_{2}\right)$ : $\delta\left(e_{i}\right)=\left\{e_{1}, e_{2}, e_{3}\right\}$, for $i=1,2,3$ (in blue on the second line right). The obtained similarity values are $s\left(e_{1}, e_{i}\right)=\frac{3}{4}, s\left(e_{2}, e_{i}\right)=\frac{3}{3}, s\left(\left\{e_{1}, e_{2}\right\} ; B\right)=\frac{3}{4}$, for $B \subseteq E_{2}, B \neq \emptyset \ldots$. (For interpretation of the references to color in this figure legend, the reader is referred to the web version of this article.)

\section{Proof.}

(a) $\forall\left(e_{i}, e_{j}\right) \in E^{1} \times E^{2}, s\left(\left(e_{i}, e_{j}\right)\right)=0 \Longleftrightarrow \forall\left(e_{i}, e_{j}\right) \in E^{1} \times E^{2}, \delta_{E^{1}}\left(e_{i}\right)$ $\cap \delta_{E^{2}}\left(e_{j}\right)=\emptyset \Rightarrow \forall\left(e_{i}, e_{j}\right) \in E^{1} \times E^{2}, e_{i} \notin \delta_{E^{2}}\left(e_{j}\right)$ and $e_{j} \notin \delta_{E^{1}}\left(e_{i}\right)$ hence $E^{1} \cap E^{2}=\emptyset$. Indeed, since $\delta_{E^{2}}$ is extensive, $e_{j} \in \delta_{E^{2}}\left(e_{j}\right)$ and $\cup_{e_{j}} \delta_{E^{2}}\left(e_{j}\right)=E^{2}$, and therefore having $e_{i} \notin \delta_{E^{2}}\left(e_{j}\right)$ for all $e_{j}$ implies $e_{i} \notin E^{2}$. Similarly $e_{j} \notin E^{1}$.

Conversely, if $E^{1} \cap E^{2} \neq \emptyset$, then $\mathcal{P}\left(E^{1}\right) \cap \mathcal{P}\left(E^{2}\right) \neq \emptyset$ and any $\delta_{E^{1}}(A)$ is disjoint from any $\delta_{E^{2}}(B)$.

(b) $\forall\left(e_{i}, e_{j}\right) \in E^{1} \times E^{2}, s\left(\left(e_{i}, e_{j}\right)\right)=1 \Longleftrightarrow \forall\left(e_{i}, e_{j}\right) \in E^{1} \times E^{2}, \delta_{E^{1}}$ $\left(e_{i}\right)=\delta_{E^{2}}\left(e_{j}\right)$. Since $\delta$ is extensive, $\forall e_{i} \in E^{1}, e_{i} \in \delta_{E^{1}}\left(e_{i}\right)$, hence $e_{i} \in \delta_{E^{2}}\left(e_{i}\right)$ and therefore $e_{i} \in E^{2}$. Similarly $\forall e_{j} \in E^{2}, e_{j} \in E^{1}$. Therefore $E^{1}=E^{2}$.

(c) The symmetry of $s$ is straightforward.

\subsection{Hypergraph kernel}

Let $X$ be a non-empty set. A function $K: X \times X \rightarrow \mathbb{R}^{+}$such that.

- $K\left(x, x^{\prime}\right)=K\left(x^{\prime}, x\right)$, for all $x, x^{\prime} \in X$,

- and $\sum_{i=1}^{n} \sum_{j=1}^{n} K\left(x_{i}, x_{j}\right) c_{i} c_{j} \geqslant 0$, for all $n \in \mathbb{N}$ and all $x_{1}, \ldots, x_{n} \in X$ and $c_{1}, \ldots, c_{n} \in \mathbb{R}$

is a positive definite kernel or kernel for short. From the properties above, an operator $K$ is a kernel if it is symmetric and its associated matrix is positive definite.

Let us consider the restriction to singletons of the similarity introduced in Definition 9:

$$
\begin{aligned}
& s: E \times E \rightarrow \mathbb{R}^{+} \\
& \left(e_{i}, e_{j}\right) \mapsto s\left(e_{i}, e_{j}\right)=\frac{\left|\delta\left(e_{i}\right) \cap \delta\left(e_{j}\right)\right|}{\left|\delta\left(e_{i}\right) \cup \delta\left(e_{j}\right)\right|}
\end{aligned}
$$

where the subscript $E$ is omitted to simplify notations. The associated matrix is $M=\left(s\left(e_{i}, e_{j}\right)\right)_{i j \in\{1 \ldots m\}}$, where $m=|E|$.

As in the previous subsection, considering overlap between dilated hyperedges provides more flexibility in the similarity measure and the kernels that will be introduced next. The similarity will be high if the hypergraphs differ, but are in better correspondence (i.e. larger overlapping of hyperedges) if a dilation is applied. The dilation then accounts for potential small differences (for instance due to some imprecision in the representation) which are considered as non significant, and can be tuned according to the application at hand. If the dilation is reduced to the identity mapping, which is a particular case, then a strict overlap is required.

If $A$ and $B$ are $m \times m$ matrices, we denote by $A \circ B$ their entrywise product, i.e. the matrix whose $m_{i, j}$ entry is $a_{i, j} b_{i, j}$. It is called the Schur product of $A$ and $B$, or the Hadamard product. It is well known that if $A$ and $B$ are positive definite, then so is $A \circ B$. This will be used in the proof of the following result.

Theorem 2. The matrix

$M=\left(s\left(e_{i}, e_{j}\right)\right)_{i, j \in\{1 \ldots m\}}$,

for $s$ defined as in Eq. (11) from a morphological dilation, is positive definite.

Proof. Let $H=(V, E)$ be a hypergraph with $E=\left\{e_{1}, \ldots, e_{m}\right\}(|E|=m)$, and $\delta:(\mathcal{P}(E), \subseteq) \rightarrow(\mathcal{P}(E), \subseteq)$ be a dilation such that $\delta(e) \neq \emptyset$ for all $e \in E$. Let $A=\left(a_{i, j}\right)_{i, j \in\{1 \ldots m\}}$ be the following matrix:

$a_{i, j}= \begin{cases}\left|\delta\left(e_{i}\right) \cap \delta\left(e_{j}\right)\right| & \text { if } i<j, \\ \left|\delta\left(e_{i}\right)\right| & \text { if } i=j, \\ -\left|\delta\left(e_{i}\right) \cap \delta\left(e_{j}\right)\right| & \text { if } i>j .\end{cases}$

This matrix can be written in the following way:

$A=\left(\begin{array}{cccccccccc}a_{1,1} & a_{1,2} & a_{1,3} & a_{1,4} & \ldots & \ldots & \ldots & \ldots & \ldots & a_{1, m} \\ -a_{1,2} & a_{2,2} & a_{2,3} & a_{2,4} & \ldots & \ldots & \ldots & \ldots & \ldots & a_{2, m} \\ -a_{1,3} & -a_{2,3} & a_{3,3} & a_{3,4} & \ldots & \ldots & \ldots & \ldots & \ldots & a_{3, m} \\ \vdots & \vdots & \ddots & \vdots & \vdots & \vdots & \vdots & \vdots & \vdots & \vdots \\ -a_{1, j} & -a_{2, j} & -a_{3, j} & -a_{3, j} & \ldots & -a_{j-1, j} & a_{j, j} & a_{j, j+1} & \ldots & a_{j, m} \\ \vdots & \vdots & \ddots & \vdots & \vdots & \vdots & \vdots & \vdots & \vdots & \vdots \\ -a_{1, m} & -a_{2, m} & -a_{3, m} & \ldots & \ldots & \ldots & \ldots & \ldots & -a_{m-1, m} & a_{m, m}\end{array}\right)$

Let $X^{t}=\left(x_{1}, x_{2}, x_{3}, \ldots, x_{m}\right)$ be a vector of $\mathbb{R}^{m}$. We have:

$$
\begin{aligned}
X^{t} A X= & \sum_{i=1}^{m} a_{1, i} x_{i} x_{1}-a_{1,2} x_{1} x_{2}+\sum_{i=2}^{m} a_{2, i} x_{i} x_{2}-\sum_{i=1}^{2} a_{i, 3} x_{i} x_{3}+\sum_{i=3}^{m} a_{3, i} x_{i} x_{3} \\
& -\sum_{i=1}^{3} a_{i, 4} x_{i} x_{4}+\sum_{i=4}^{m} a_{4, i} x_{i} x_{4}-\cdots-\sum_{i=1}^{j-1} a_{i, j} x_{i} x_{j}+\sum_{i=j}^{m} a_{j, i} x_{i} x_{j}-\cdots \\
& -\sum_{i=1}^{m-1} a_{i, m-1} x_{i} x_{m}+a_{m, m} x_{m}^{2} \\
= & a_{1,1} x_{1}^{2}-a_{1,2} x_{1} x_{2}+\sum_{i=2}^{m} a_{1, i} x_{i} x_{1}+a_{2,2} x_{2}^{2}+\sum_{i=3}^{m} a_{2, i} x_{i} x_{2} \\
& -\sum_{i=1}^{2} a_{i, 3} x_{i} x_{3}+a_{3,3} x_{3}^{2}+\sum_{i=4}^{m} a_{3, i} x_{i} x_{3}+\cdots+a_{j, j} x_{j}^{2}+\sum_{i=j+1}^{m} a_{j, i} x_{i} x_{j}-\cdots \\
& -\sum_{i=1}^{m-1} a_{i, m-1} x_{i} x_{m}+a_{m, m} x_{m}^{2} \\
= & \sum_{i=1}^{m} a_{i, i} x_{i}^{2}-\sum_{i=2}^{m} a_{1, i} x_{1} x_{i}+\sum_{i=2}^{m} a_{1, i} x_{i} x_{1}-\sum_{i=3}^{m} a_{2, i} x_{2} x_{i}+\sum_{i=3}^{m} a_{2, i} x_{i} x_{2} \\
& +\cdots-\sum_{i=j+1}^{m} a_{j, i} x_{j} x_{i}+\sum_{i=j+1}^{m} a_{j, i} x_{i} x_{j}+\cdots-a_{m-1, m-1} x_{m-1} x_{m} \\
& +a_{m-1, m-1} x_{m} x_{m-1} \\
= & \sum_{i=1}^{m} a_{i, i} x_{i}^{2}>0 .
\end{aligned}
$$


Consequently the matrix $A$ is positive definite.

Let $B=\left(b_{i, j}\right)_{i, j \in\{1, \ldots, m\}}$ be the following matrix:

$b_{i, j}= \begin{cases}\frac{1}{\left|\delta\left(e_{i}\right) \cup \delta\left(e_{j}\right)\right|} & \text { if } i<j, \\ \frac{1}{\left|\delta\left(e_{i}\right)\right|} & \text { if } i=j, \\ -\frac{1}{\left|\delta\left(e_{i}\right) \cup \delta\left(e_{j}\right)\right|} & \text { if } i>j .\end{cases}$

This matrix can be written in a similar form as the matrix $A$. Hence $X^{t} B X=\sum_{i=1}^{m} b_{i, i} x_{i}^{2}>0$. So $B$ is positive definite.

It is easy to verify that $M=A \circ B$. We can conclude that $M$ is a positive definite matrix.

Corollary 2. The similarity defined by $s\left(e_{i}, e_{j}\right)=\frac{\left|\delta\left(e_{i}\right) \cap \delta\left(e_{j}\right)\right|}{\delta\left(e_{i}\right) \cup \delta\left(e_{i}\right) \mid}$ is a kernel. If $A$ is an $m \times n$ matrix and $B$ is a $p \times q$ matrix, then the Kronecker product $A \otimes B$ is the $m p \times n q$ block matrix

$\mathbf{A} \otimes \mathbf{B}=\left(\begin{array}{ccc}a_{11} B & \cdots & a_{1 n} B \\ \vdots & \ddots & \vdots \\ a_{m 1} B & \cdots & a_{m n} B\end{array}\right)$

The Kronecker product of matrices corresponds to the abstract tensor product of linear mappings. It is well known that if $A$ and $B$ are positive definite matrices, then so is $A \otimes B$.

Now let us consider two dilations $\delta$ and $\delta^{\prime}$ defined on $(\mathcal{P}(E), \subseteq)$ and $\left(\mathcal{P}\left(E^{\prime}\right), \subseteq\right)$, respectively, and the associated similarity functions:

$s: E \times E \rightarrow \mathbb{R}^{+}$

$\left(e_{i}, e_{j}\right) \mapsto s\left(e_{i}, e_{j}\right)=\frac{\left|\delta\left(e_{i}\right) \cap \delta\left(e_{j}\right)\right|}{\left|\delta\left(e_{i}\right) \cup \delta\left(e_{j}\right)\right|}$

and

$s^{\prime}: E^{\prime} \times E^{\prime} \rightarrow \mathbb{R}^{+}$

$\left(e_{i}^{\prime}, e_{j}^{\prime}\right) \mapsto s^{\prime}\left(e_{i}^{\prime}, e_{j}^{\prime}\right)=\frac{\left|\delta^{\prime}\left(e_{i}^{\prime}\right) \cap \delta^{\prime}\left(e_{j}^{\prime}\right)\right|}{\left|\delta^{\prime}\left(e_{i}^{\prime}\right) \cup \delta^{\prime}\left(e_{j}^{\prime}\right)\right|}$

defining the two matrices $M=\left(s\left(e_{i}, e_{j}\right)\right)_{i j \in\{1 \ldots m\}}$ and $M^{\prime}=\left(s^{\prime}\left(e_{i}^{\prime}, e_{j}^{\prime}\right)\right)_{i, j \in\left\{1, \ldots, m^{\prime}\right\}}$, with $m=|E|$ and $m^{\prime}=\left|E^{\prime}\right|$.

Hence $M^{\prime \prime}=M \otimes M^{\prime}$ defines a kernel. So we have the following result:

\section{Proposition 13. The mapping}

$$
\begin{aligned}
& s^{\prime \prime}:(E \times E) \times\left(E^{\prime} \times E^{\prime}\right) \rightarrow \mathbb{R}^{+} \\
& \left(\left(e_{i}, e_{j}\right) ;\left(e_{k}^{\prime}, e_{l}^{\prime}\right)\right) \mapsto s^{\prime \prime}\left(\left(e_{i}, e_{j}\right) ;\left(e_{k}^{\prime}, e_{l}^{\prime}\right)\right)=s\left(e_{i}, e_{j}\right) s^{\prime}\left(e_{k}^{\prime}, e_{l}^{\prime}\right)
\end{aligned}
$$

defines a kernel.

This kernel provides a comparison measure between two hypergraphs or substructures of hypergraphs. This measure can be computed with a polynomial time complexity in the number of hyperedges. Interesting applications could be for instance the comparison of sub-molecules between two molecules when they are modeled by hypergraphs, or the comparison of two scenes in images when they are again modeled by hypergraphs. A typical example would be to consider vertices as image regions or objects, and hyperedges representing binary or n-ary relations between them (such as spatial relations). The two images to be compared could be a model and a specific image, two images where changes may occur, as in video sequences, or two images representing different instances of a phenomena (such as medical images of different patients). Such examples have raised a lot of literature on graph-based methods for solving recognition problems, but very little using hypergraphs. Providing comparison tools is then a first step towards this aim.

\section{Morphological metric on hypergraphs}

In this section we again consider a hypergraph $H=(V, E)$, a dilation $\delta$ defined on $(\mathcal{P}(E), \subseteq)$, and its restriction on singletons to derive a metric on the hyperedges of $H$.

Proposition 14. Let $H=(V, E)$ be a hypergraph equipped with a dilation $\delta$ on $(\mathcal{P}(E), \subseteq)$ that is assumed to be injective on $E$ $\left(\forall\left(e, e^{\prime}\right) \in E^{2}, \quad \delta(e)=\delta\left(e^{\prime}\right) \Rightarrow e=e^{\prime}\right)$. Then $\tilde{s}\left(e, e^{\prime}\right)=1-s\left(e, e^{\prime}\right)$ is a metric, where $s$ is defined from $\delta$ as in Eq. (11).

Proof. Clearly $\forall\left(e, e^{\prime}\right) \in E^{2}, \tilde{s}\left(e, e^{\prime}\right) \geqslant 0$, and $\tilde{s}$ is symmetrical.

As for the separation axiom, we have: $\tilde{s}\left(e, e^{\prime}\right)=0 \Rightarrow s\left(e, e^{\prime}\right)=$ $1 \Rightarrow \delta(e)=\delta\left(e^{\prime}\right) \Rightarrow e=e^{\prime}$; the converse is obvious.

Let us now consider the triangular inequality. We have:

$$
\begin{aligned}
\tilde{s}\left(e, e^{\prime}\right) & =1-\frac{\left|\delta(e) \cap \delta\left(e^{\prime}\right)\right|}{\left|\delta(e) \cup \delta\left(e^{\prime}\right)\right|}=\frac{\left|\delta(e) \cup \delta\left(e^{\prime}\right)\right|-\left|\delta(e) \cap \delta\left(e^{\prime}\right)\right|}{\left|\delta(e) \cup \delta\left(e^{\prime}\right)\right|} \\
& =\frac{\left|\delta(e) \triangle \delta\left(e^{\prime}\right)\right|}{\left|\delta(e) \cup \delta\left(e^{\prime}\right)\right|} .
\end{aligned}
$$

The mapping $d: X \times X \rightarrow \mathbb{R}^{+}$with $d\left(e, e^{\prime}\right)=\left|\delta(e) \triangle \delta\left(e^{\prime}\right)\right|$ is a metric.

Let $(X ; d)$ be a metric space and $a \in X$ then $D(x, y)=$ $\frac{2 d(x, y)}{d(x, a)+d(y, a)+d(x, y)}$ is a metric on $X$.

Now: $\quad D\left(e, e^{\prime}\right)=\frac{2\left|\delta(e) \Delta \delta\left(e^{\prime}\right)\right|}{|\delta(\boldsymbol{e}) \Delta \theta|+\left|\phi \Delta \delta\left(e^{\prime}\right)\right|+\left|\delta(\boldsymbol{e}) \Delta \delta\left(e^{\prime}\right)\right|}=\frac{2\left|\delta(e) \Delta \delta\left(e^{\prime}\right)\right|}{2\left|\delta(\boldsymbol{e}) \cup \delta\left(e^{\prime}\right)\right|}=\tilde{\boldsymbol{s}}\left(e, e^{\prime}\right)$. So $\tilde{s}\left(e, e^{\prime}\right)$ is a metric called morphological metric.

It is interesting to note that this distance involves the symmetrical difference between dilations of hyperedges. It could be used to search patterns or regularities in a hypergraph, which can be very useful in chemoinformatic for instance. In image understanding, it could be used to extract common information (common parts, regions or objects) in one or several images, based on their hypergraph representations. For instance if an image presents several instances of similar objects, a hypergraph representation could represent the subparts of the objects as vertices and an object as a hyperedge linking all its subparts. Finding similar hyperedges would then allow extracting all objects of the same nature from the image. Dilations can then be useful to increase robustness in the case where imprecision occurs in the definition of objects subparts, or to take also neighborhood regions (i.e. the local context of each object) into account in the comparison. Distances can also be used as relevant features in kernel based methods for classification and recognition.

\section{A simple illustrative example}

As an example on images, we consider a simple case, where a hypergraph is built from the elementary connectivity relation on a binary image and the associated simplicial complex. Let us assume that the 8 -connectivity is considered on a 2D image. The pixel connectivity graph is $\Gamma$ where the vertices are the image pixels and the set of edges is associated with the connectivity relation (i.e. any two 8-connected pixels in the image form an edge). This graph generates a simplicial complex 5 :

- the set of vertices of $\Gamma$ is the set of simplices with a dimension $d=0$,

- the maximal cliques of $\Gamma$ is the set of simplices with a dimension $d \geqslant 1$.

\footnotetext{
${ }^{5}$ A simplicial complex is a collection of subsets $\mathcal{S}$ of a set $X=\left\{v_{1}, \ldots, v_{n}\right\}$ such that if $\sigma \in \mathcal{S}$ and $\tau \subseteq \sigma$ then $\tau \in \mathcal{S}$. An element $\sigma \in \mathcal{S}$ is called a simplex of dimension $|\sigma|-1$ and a subset of a simplex is called a face. A facet is a $k-1$ dimensional face of a $k$-dimensional simplex. The dimension of a simplicial complex is the maximal dimension of its simplices.
} 


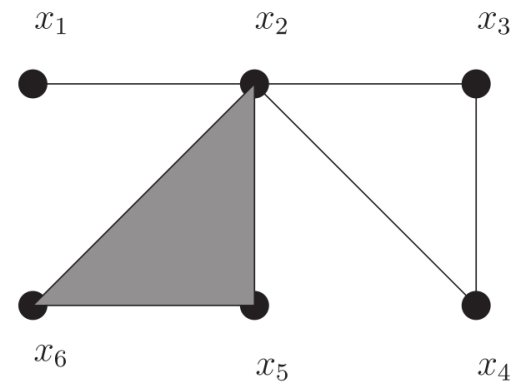

Fig. 8. Simplicial complex, in which the simplices of dimension $d=0$ are $\left\{x_{1}\right\},\left\{x_{2}\right\}$, $\left\{x_{3}\right\},\left\{x_{4}\right\},\left\{x_{5}\right\},\left\{x_{6}\right\}$, the simplices of dimension $d=1$ are $\left\{x_{1}, x_{2}\right\},\left\{x_{2}, x_{3}\right\},\left\{x_{2}, x_{4}\right\}$, $\left\{x_{2}, x_{5}\right\},\left\{x_{2}, x_{6}\right\},\left\{x_{3}, x_{4}\right\},\left\{x_{5}, x_{6}\right\}$, and the simplices of dimension $d=2$ are $\left\{x_{2}, x_{5}, x_{6}\right\}$.

Hence this simplicial complex has a dimension $d \leqslant 3$ and $\Gamma$ is the underlying graph. We associate a hypergraph $H=(V, E)$ with this simplicial complex as follows:

- $V$ is the set of simplices with a dimension $d=0$;

- $E$ is the set of maximal simplices with a dimension $d \geqslant 1$.

For instance the hypergraph $H=(V, E)$ associated with the simplicial complex from Fig. 8 is:

- $V=\left\{\left\{x_{1}\right\},\left\{x_{2}\right\},\left\{x_{3}\right\},\left\{x_{4}\right\},\left\{x_{5}\right\},\left\{x_{6}\right\}\right\}$;

- $E=\left\{\left\{x_{1}, x_{2}\right\},\left\{x_{2}, x_{3}\right\},\left\{x_{2}, x_{4}\right\},\left\{x_{3}, x_{4}\right\},\left\{x_{2}, x_{5}, x_{6}\right\}\right\}$.

Let us now give an example of morphological dilation on such a simplicial simplex representing an image as a hypergraph $H=(V, E)$. Let us consider a dilation $\delta$ on $(\mathcal{P}(E), \subseteq)$, defined on singletons as:

$\forall e \in E, \delta(e)=\left\{e^{\prime} \in E \mid v(e) \cap v\left(e^{\prime}\right) \neq \emptyset\right\}$,

and for any subset:

$\forall A \subseteq E, \delta(A)=\cup_{e \in A} \delta(\{e\})$.

This dilation is the one given in Example 1 in Section 4.3. For any $A$, $\delta(A)$ defines a partial hypergraph $(V, \delta(A))$ of $H$, which corresponds to a unique subcomplex. This dilation is illustrated in Fig. 9.

Let us now consider a dilation similar to the one introduced in Example 2 in Section 4.3, with a constraint on the cardinality of hyperedges, for $k>1$ :

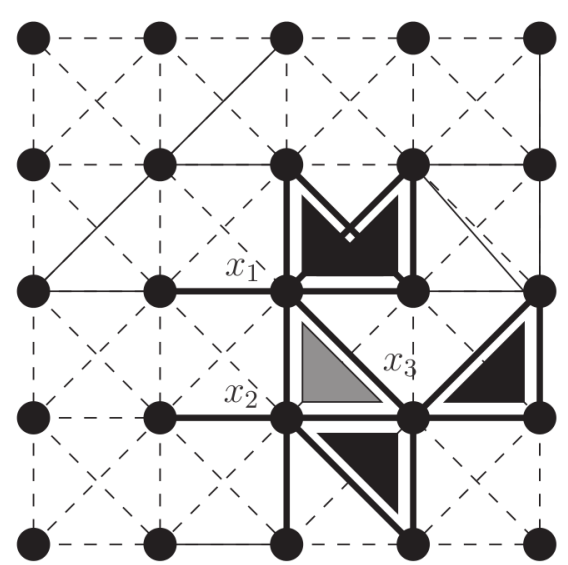

Fig. 9. A simple image equipped with the 8-connectivity relation. A partial hypergraph and a subcomplex are represented by thin lines, thick lines and triangles. The subcomplex generated by the dilation, defined in Eqs. (12) and (13) of the hyperedge $\left\{x_{1}, x_{2}, x_{3}\right\}$ is given by the thick lines, triangles and the vertices incident to the thick lines.

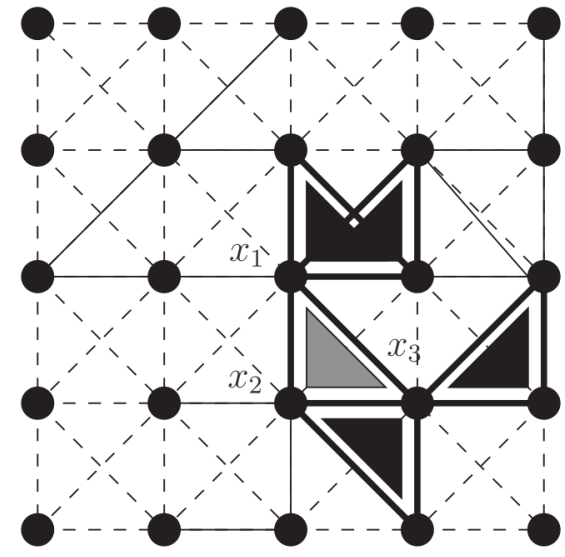

Fig. 10. As in Fig. 9, a partial hypergraph and a subcomplex are represented by thin lines, thick lines and triangles. The subcomplex generated by the dilation, defined in Eqs. (14) and (15) for $k=3$, of the hyperedge $\left\{x_{1}, x_{2}, x_{3}\right\}$ is given by triangles, thick lines and the vertices incident to these thick lines.

$\forall e \in E, \delta(e)=\left\{e^{\prime} \in E \mid v(e) \cap v\left(e^{\prime}\right) \neq \emptyset\right.$ and $\left.\left|e^{\prime}\right|=k\right\}$,

and

$\forall A \subseteq E, \delta(A)=\cup_{e \in A} \delta(\{e\})$.

This dilation is illustrated in Fig. 10. The dilation of the hyperedge $\left\{x_{1}, x_{2}, x_{3}\right\}$ is provided for $k=3$.

Defining morphological operators on simplicial complexes has also been addressed in [21], relying on the same complete lattices. Other forms of dilations were proposed, based on closure and star operators, and considering the smallest complex which contains a subset $X$, or the largest complex contained in $X$, respectively. Adjoint erosions were derived, as well as granulometries. Operators acting on complexes of fixed dimensions were proposed too. Applications included alternate sequential filters on simplicial complexes derived from 6-connectivity on a hexagonal grid, and mesh filtering.

\section{Conclusion}

In this work we introduced mathematical morphology on hypergraphs. We proposed several lattices built on hypergraphs, the most interesting ones being when the structure of the hypergraphs is actually involved in the partial ordering. We then derived morphological operators on these lattices, and provided several concrete examples. One of them exhibits a particular dilation which characterizes the transversals of a hypergraph. To show the relevance of the relationship between the two domains of mathematical morphology and of hypergraphs, we have exhibited a notion of duality in mathematical morphology which corresponds to the concept of duality which is important in the theory of hypergraphs. Other properties of hypergraphs can undoubtedly be expressed using morphological operators, such as matching contained in a subset of vertices of a hypergraph. We are currently investigating how to use mathematical morphology to improve some results on hypergraphs.

A notion of similarity of hypergraphs was also introduced via morphological operators. This allowed us to define a positive kernel on hypergraphs. From similarity an original notion of distance between hyperedges is derived. We are also currently investigating how to compare this topology with those defined in [32,33]. Other definitions of similarities between hypergraphs having different sets of vertices have also to be investigated. In this paper, similarities were defined from dilations. They could also be defined from 
other morphological operators, in particular closing, which would bring additional filtering properties.

As an extension of the proposed framework and of the example provided on a simple image, applications to image analysis and classification can be foreseen from the concepts defined in this article, and this will be addressed in our future work.

\section{Acknowledgment}

This work was partially funded by a grant from Institut MinesTélécom/Télécom ParisTech, and was initiated during the sabbatical stay of A. Bretto at Télécom ParisTech.

\section{References}

[1] J. Serra, Image Analysis and Mathematical Morphology, Academic Press, New York, 1982.

[2] J. Serra (Ed.), Image Analysis and Mathematical Morphology, Part II: Theoretical Advances, Academic Press, London, 1988.

[3] P. Soille, Morphological Image Analysis, Springer Verlag, Berlin, 1999.

[4] L. Najman, H. Talbot (Eds.), Mathematical Morphology: From Theory to Applications, ISTE-Wiley, 2010.

[5] H.J.A.M. Heijmans, Morphological Image Operators, Academic Press, Boston, 1994.

[6] H.J.A.M. Heijmans, C. Ronse, The Algebraic Basis of Mathematical Morphology - Part I: Dilations and Erosions, Computer Vision, Graphics and Image Processing 50 (3) (1990) 245-295.

[7] C. Ronse, Why mathematical morphology needs complete lattices, Signal Processing 21 (2) (1990) 129-154.

[8] C. Ronse, H.J.A.M. Heijmans, The algebraic basis of mathematical morphology Part II: openings and closings, Computer Vision, Graphics and Image Processing 54 (1) (1991) 74-97.

[9] R. Keshet, Mathematical morphology on complete semilattices and its applications to image processing, Fundamenta Informaticae 41 (1-2) (2000) $33-56$.

[10] C. Berge, Hypergraphs, Elsevier Science Publisher, The Netherlands, 1989.

[11] V.I. Voloshin, Introduction to Graph and Hypergraph Theory, Nova Science, 2009.

[12] A. Bretto, J. Azema, H. Cherifi, B. Laget, Combinatorics and image processing, CVGIP: Graphical Model and Image Processing 59 (5) (1997) 265-277.

[13] A. Bretto, H. Cherifi, D. Aboutajdine, Hypergraph imaging: an overview, Pattern Recognition 35 (3) (2002) 651-658.
[14] S. Rital, Hypergraph cuts \& unsupervised representation for image segmentation, Fundamenta Informaticae 96 (1-2) (2009) 153-179.

[15] A. Ducournau, A. Bretto, S. Rital, B. Laget, A reductive approach to hypergraph clustering: an application to image segmentation, Pattern Recognition 45 (7) (2012) 2788-2803.

[16] Q. Liu, Y. Huang, D. Metaxas, Hypergraph with sampling for image retrieval, Pattern Recognition 44 (10-11) (2011) 2255-2262.

[17] J. Cousty, L. Najman, J. Serra, Some morphological operators in graph spaces, in: M. Wilkinson, J. Roerdink (Eds.), International Symposium on Mathematical Morphology (ISMM 2009), LNCS, vol. 5720, 2009, pp. 149-160.

[18] F. Meyer, J. Stawiaski, Morphology on graphs and minimum spanning trees, in: M. Wilkinson, J. Roerdink (Eds.), International Symposium on Mathematical Morphology (ISMM 2009), LNCS, vol. 5720, 2009, pp. 161-170.

[19] V.-T. Ta, A. Elmoataz, O. Lezoray, Partial difference equations over graphs: morphological processing of arbitrary discrete data, in: European Conference on Computer Vision (ECCV 2008), LNCS, vol. 5304, 2008, pp. 668-680.

[20] L. Vincent, Graphs and mathematical morphology, Signal Processing 16 (4) (1989) 365-388.

[21] F. Dias, J. Cousty, L. Najman, Some morphological operators on simplicial complex spaces, in: Discrete Geometry for Computer Imagery (DGCI 2011), LNCS, vol. 6607, Nancy, France, 2011, pp. 441-452.

[22] L. Zager, Graph Similarity and Matching, Ph.D. thesis, MIT Department of Electrical Engineering and Computer Science, 2005.

[23] B. Schölkopf, A. Smola, Learning with Kernels, MIT Press, Cambridge, MA, 2002.

[24] I. Bloch, A. Bretto, Mathematical morphology on hypergraphs: preliminary definitions and results, in: Discrete Geometry for Computer Imagery (DGCl 2011), LNCS, vol. 6607, Nancy, France, 2011, pp. 429-440.

[25] I. Bloch, H. Heijmans, C. Ronse, Mathematical morphology, in: M. Aiello, I. Pratt-Hartman, J. van Benthem (Eds.), Handbook of Spatial Logics, Springer, 2007, pp. 857-947 (chapter 13).

[26] T. Eiter, G. Gottlob, Identifying the minimal transversals of a hypergraph and related problems, SIAM Journal on Computing 24 (6) (1995) 1278-1304.

[27] I. Bloch, On links between mathematical morphology and rough sets, Pattern Recognition 33 (9) (2000) 1487-1496.

[28] J. Stell, Relational granularity for hypergraphs, in: Rough Sets and Current Trends in Computing, LNAI, vol. 6086, 2010, pp. 267-276.

[29] S. Klamt, U.-U. Haus, F. Theis, Hypergraphs and cellular networks, PLoS Computational Biology 5 (5) (2009) el000385.

[30] M. Yang, Y. Yang, A hypergraph approach to linear network coding in multicast networks, IEEE Transactions on Parallel and Distributed Systems 21 (7) (2010) 968-982.

[31] L. Zhen, Z. Jiang, Hy-SN: hyper-graph based semantic network, KnowledgeBased Systems 23 (8) (2010) 809-816.

[32] A. Bretto, Comparability graphs and digital topology, Computer Vision and Image Understanding 82 (1) (2001) 33-41.

[33] T.Y. Kong, A. Rosenfeld, Digital topology: introduction and survey, Computer Vision, Graphics, and Image Processing 48 (3) (1989) 357-393. 